TRANSACTIONS OF THE

AMERICAN MATHEMATICAL SOCIETY

Volume 361, Number 1, January 2009, Pages 495-523

S 0002-9947(08)04534-0

Article electronically published on June 26, 2008

\title{
LIFTABLE DERIVATIONS FOR GENERICALLY SEPARABLY ALGEBRAIC MORPHISMS OF SCHEMES
}

\author{
ROLF KÄLLSTRÖM
}

\begin{abstract}
We consider dominant, generically algebraic (e.g. generically finite), and tamely ramified (if the characteristic is positive) morphisms $\pi$ : $X / S \rightarrow Y / S$ of $S$-schemes, where $Y, S$ are Nœtherian and integral and $X$ is a Krull scheme (e.g. normal Nœtherian), and study the sheaf of tangent vector fields on $Y$ that lift to tangent vector fields on $X$. We give an easily computable description of these vector fields using valuations along the critical locus. We apply this to answer the question when the liftable derivations can be defined by a tangency condition along the discriminant. In particular, if $\pi$ is a blow-up of a coherent ideal $I$, we show that tangent vector fields that preserve the Ratliff-Rush ideal (equals $\left[I^{n+1}: I^{n}\right]$ for high $n$ ) associated to $I$ are liftable, and that all liftable tangent vector fields preserve the integral closure of $I$. We also generalise in positive characteristic Seidenberg's theorem that all tangent vector fields can be lifted to the normalisation, assuming tame ramification.
\end{abstract}

\section{INTRODUCTION}

Let $\pi: X / S \rightarrow Y / S$ be a dominant morphism of $S$-schemes, where $Y$ and $S$ are Nœtherian and $X$ is a Krull scheme (e.g. Nœtherian and normal), so in particular $X$ and $Y$ are integral. Consider the diagram

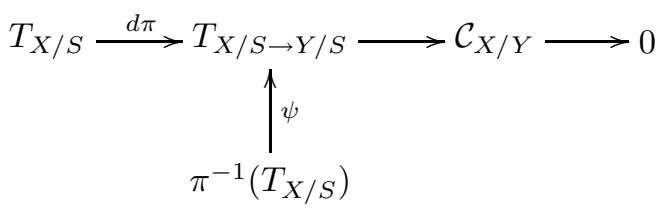

where $T_{X / S}=\operatorname{Hom}_{\mathcal{O}_{X}}\left(\Omega_{X / S}, \mathcal{O}_{X}\right)$ is the sheaf of $S$-relative tangent vector fields, $d \pi$ is the tangent morphism and $\psi$ is the canonical morphism to the sheaf $T_{X / S \rightarrow Y / S}$ of derivations from $\pi^{-1}\left(\mathcal{O}_{Y}\right)$ to $\mathcal{O}_{X}$; if $Y / S$ is locally of finite type and either $\pi$ is flat or $Y / S$ is smooth, then $T_{X / S \rightarrow Y / S}=\pi^{*}\left(T_{Y / S}\right)$. The critical locus $C_{\pi}$ is the support of the critical module $\mathcal{C}_{X / Y}$, and the discriminant set $D_{\pi}$ is the closure of $\pi\left(C_{\pi}\right)$. We say that a section $\partial$ of $T_{Y / S}$ is liftable if there exists a section $\bar{\partial}$ of $T_{X / S}$ such that $d \pi(\bar{\partial})=\psi(\partial)$ (suppressing domains of definition of $\partial$ and $\bar{\partial}$ ). We denote the sheaf of liftable tangent vector fields by $T_{Y / S}^{\pi}$.

Received by the editors November 22, 2006 and, in revised form, April 13, 2007.

2000 Mathematics Subject Classification. Primary 14E22, 13N15; Secondary 14Axx, 13B22, $16 \mathrm{~W} 60$.

(C)2008 American Mathematical Society Reverts to public domain 28 years from publication 
Let $T_{Y / S}\left(\log ^{\pi} I_{D_{\pi}}\right)=T_{Y / S}\left(\log ^{\pi} D_{\pi}\right)$ be the sheaf of $\pi$-logarithmic vector fields. This sheaf is easily computed using the discrete valuations $\nu_{1}, \ldots, \nu_{r}$ of the function field of $X$ defined by order of vanishing along the components of the critical divisor locus $C_{\pi}=C_{1}+C_{2}+\cdots+C_{r}$. A section $\partial$ in $T_{Y / S}$ is $\pi$-logarithmic, or logarithmic along the morphism $\pi$, if it does not lower the value for the discrete valuations at the generic points of $C_{\pi}$ of local generators of the ideal $I_{D_{\pi}}$, at points below the generic points, i.e. $\nu_{i}\left(\partial\left(f_{j}\right)\right) \geq \nu_{i}\left(f_{j}\right)$ when $f_{j} \in I_{i}$, where $I_{i}$ is any defining ideal of the discriminant $D_{\pi}$ at the generic point of $\pi\left(C_{i}\right)$. The sheaf of logarithmic vector fields along a subscheme defined by an ideal $I$, denoted $T_{Y / S}(I)$, is the sheaf of tangent vector fields $\partial$ that preserve the ideal $I, \partial(I) \subset I$.

We describe the liftable tangent vector fields when $\pi$ is generically algebraic (the induced extension of function fields is algebraic), and tamely ramified (Def. 1.5.5).

Theorem 2.2.1, Let $\pi: X / S \rightarrow Y / S$ be a morphism of schemes over a scheme $S$. Assume that $Y$ and $S$ are Noetherian and $X$ is a Krull scheme. Assume that $\pi$ is dominant, generically algebraic and tamely ramified. If $Y$ is of unequal characteristic, assume that $Y$ is absolutely tamely ramified. Let $D_{\pi}$ be the discriminant set of $\pi$. Then:

$\left(L_{1}\right)$ Let $J_{D_{\pi}}$ be any defining ideal of $D_{\pi}$. Then

$$
T_{Y / S}^{\pi}=T_{Y / S}\left(\log ^{\pi} J_{D_{\pi}}\right) .
$$

$\left(L_{2}\right)$ If the residue field extension $k_{c} / k_{\pi(c)}$ is algebraic for each point $c \in C_{\pi}$ of height 1 , then

$$
T_{Y / S}^{\pi}=T_{Y / S}\left(I_{D_{\pi}}\right)
$$

where $I_{D_{\pi}}$ is the (reduced) ideal of the closure of the discriminant set $D_{\pi}$.

$\left(L_{3}\right)$ Let $I_{C_{\pi}}$ be the ideal of the critical locus of $\pi$. Then $\pi^{-1}\left(T_{Y / S}^{\pi}\right) \subset T_{X / S}\left(I_{C_{\pi}}\right)$; i.e. the liftable vector fields are tangent to the critical locus.

We give concrete examples at the end of the paper.

Here $\left(L_{1}\right)$ is the main new content of Theorem 2.2.1 while $\left(L_{2}\right)$ is a generalisation of earlier work, but then usually formulated for affine schemes. The first result of this type is due to Zariski who proved the following ([36, $\S 5$, Theorem 2). Let $R$ be an integrally closed local Nagata ring, with quotient field $K(R)$. Let $R^{\prime}$ be the integral closure in a finite separable extension $L$ of $K(R)$ (thus $R^{\prime}$ is finite over $R$ ). Let $D \subset \operatorname{Spec} R$ be the set of ramified primes of height 1 in $R$, and assume that each prime in $D$ is tamely ramified. Let $\partial$ be a derivation of $R$ such that for each point $x \in D$ of height 1 there exists an element $r \in \mathfrak{m}_{x} \backslash \mathfrak{m}_{x}^{2}$ such that $\partial(r)=0$. It then follows that $\partial\left(R^{\prime}\right) \subset R^{\prime}$. According to Theorem 2.2.1, with $X=\operatorname{Spec} R^{\prime}$ and $Y=\operatorname{Spec} R$, knowing the existence of the element $r$ is not necessary, as is the assumption that $R$ be a Nagata ring; the sufficient and necessary condition is $\left(L_{2}\right)$. Scheja and Storch [29] proved $\left(L_{2}\right)$ when $R$ contains the rational numbers, assuming the $R$-module $R \partial(R)$ to be of finite type. We see that this assumption is not needed and we also get the assertion in positive characteristic when $R / A$ is tamely ramified.

A dominant morphism $\pi$ is weakly submersive if $T_{Y / S}^{\pi}=T_{Y / S}$; i.e. all tangent vector fields lift to vector fields on $X$. If $Y / S$ is non-smooth and $\pi$ is non-flat, this is not the same as the surjective tangent morphism $d \pi$, in which case we say $\pi$ is submersive. Seidenberg [30] proved that the normalisation morphism of 
a Nœtherian integral scheme $X / S$ is weakly submersive when $S$ is defined over the rational numbers. We generalise this to positive characteristic (Th. 2.3.1), requiring that the normalisation morphism be tamely ramified. The proof is by reducing to the case when the normalisation is a discrete valuation ring, which is quite different from Seidenberg's proof (see Remark 2.3.4). In a forthcoming paper we will prove that the constructive resolutions of singularities of a variety $X / k$ $($ Char $k=0)$ presented in [4,8,35] are weakly submersive. This also gives the result that the multiplier ideal $J(\alpha)$ (discussed in [20) of a coherent ideal $I$ is preserved by derivations that preserve $I$; i.e. $T_{X / k}(I) \subset T_{X / k}(J(\alpha))$.

Theorem 2.2.1 describes the liftable derivations at generic points of the discriminant $D_{\pi}$ in terms of a set of discrete valuations associated to the critical locus. A natural question is whether the liftable tangent fields coincide with the vector fields that are tangent to some subscheme whose underlying space is $D_{\pi}$. More precisely, does there exist a coherent defining ideal $I$ of $D_{\pi}$ satisfying

$$
T_{Y / S}^{\pi}=T_{Y / S}(I) ?
$$

The best one can hope for, second to weakly submersive, is that all vector fields that are tangent to the discriminant can be lifted; i.e. the above equality holds when $I$ is the (radical) ideal of $D_{\pi}$. We call such morphisms differentially ramified, and know already from Theorem 2.2.1 that residually algebraic tamely ramified morphisms are differentially ramified. A stronger notion is that $\pi$ be uniformly ramified, meaning that at each generic point $\xi$ of the critical locus $C_{\pi}$, the stalk $I_{D_{\pi}, \pi(\xi)}$ has a basis with constant value in the valuation ring $\mathcal{O}_{X, \xi}$. We prove in Theorem 3.1 .3 that uniformly ramified morphisms are differentially ramified, and if for each generic point $\xi \in C_{\pi}$ there exists a basis $\left\{x_{1}, \ldots, x_{r}\right\}$ of $I_{D, \pi(\xi)}$ and derivations $\left\{\partial_{1}, \ldots, \partial_{r}\right\} \subset T_{Y / S, \pi(\xi)}$ such that the matrix $\left(\partial_{j}\left(x_{i}\right)\right)$ is invertible, then the converse also holds.

It is particularly interesting to characterise the liftable tangent fields for birational morphisms, forming an important class of generically separably algebraic morphisms. In the light of $\left(L_{2}\right)$ and $\left(L_{3}\right)$ in Theorem 2.2.1 this also characterises the liftable vector fields for "alterations", i.e. compositions of finite and birational morphisms. We have already discussed certain birational morphisms, but now consider any (projective) birational morphism of integral Notherian schemes, which we know always is the blow-up of some fractional ideal. Hence let $\pi: B l_{I}(X) \rightarrow X$ be the blow-up of a given fractional ideal $I$ on $X$. Here $B l_{I}(X)$ need not be Krull (normal), so Theorem 2.2.1 is not directly applicable, but we can apply it to attain a lower and upper inclusion for $T_{X / S}^{\pi}$ by sheaves of vector fields that are tangent to certain subschemes with the same underlying space as the discriminant. One first easily gets that the sub-Lie algebroid of vector fields that preserve $I$ is liftable, $T_{X / S}(I) \subset T_{X / S}^{\pi}$, but in general this inclusion is strict. There is also a latitude in the choice of $I$, for different ideals may give the same blow-up, and we ask if there is a choice $\tilde{I}$ such that $B l_{I}(X) \cong B l_{\tilde{I}}(X)$ (isomorphism over $\left.X\right)$ and $T_{X / S}^{\pi}=T_{X / S}(\tilde{I})$ ? Let $\hat{I}=\bigcup_{n>1}\left[I^{n+1}: I^{n}\right]$ be the Ratliff-Rush ideal associated to $I$ [27] and $\bar{I}$ its integral closure; then $I \subset \hat{I} \subset \bar{I}$. In Theorem 3.2 .2 we prove

$$
T_{X / S}(\hat{I}) \subset T_{X / S}^{\pi} \subset T_{X / S}(\bar{I}),
$$

where the right-hand inclusion holds under the additional assumption that the normalisation morphism of $B l_{I}(X)$ be tamely ramified (make this assumption in 
this paragraph). We therefore succeed in a positive answer to our question $(*)$ above when the Ratliff-Rush ideal $\hat{I}$ associated to $I$ is integrally closed, getting $T_{X / S}^{\pi}=$ $T_{X / S}(\hat{I})$, since $B l_{\hat{I}}(X)$ is isomorphic to $B l_{I}(X)$. An immediate consequence is that blow-ups of radical ideals are differentially ramified. Combining with Theorem 3.1.3 one also gets this: Let $\pi: \tilde{X} / S \rightarrow X / S$ be a tamely ramified blow-up of a reduced subscheme $V / S$ where $\tilde{X}$ is assumed to be Krull and $X / S$ is a Notherian integral scheme which is smooth at the generic points of $V$. Then $\pi$ is uniformly ramified (Cor. 3.2.3).

One should note that several results in this paper have straightforward holomorphic counterparts. For instance, for a finite holomorphic map $\pi: \mathbf{C}^{m} \rightarrow \mathbf{C}^{m}$ we have $T_{\mathbf{C}^{m}}^{\pi}=T_{\mathbf{C}^{m}}\left(I_{D_{\pi}}\right)$, which was proven in a different way in [2].

We also mention how liftable tangent vector fields can be used. One may interpret $T_{Y / S}^{\pi}$ as the sheaf of infinitesimal symmetries of $\pi$, so that the fibres of liftable tangent vector fields correspond to directions in the base where the fibres of $\pi$ do not deform. Thus a good understanding of $T_{Y / S}^{\pi}$ is useful for the study of deformations in a (flat) family of schemes. We tie this up with Zariski's notion of analytic equisingularity stratification of a hypersurface $X$ in $\mathbf{C}^{n}$. The dominant stratum is where $X$ is smooth, so that smooth points do not belong to the critical locus of the restriction $\pi=\left.p\right|_{X}: X \rightarrow \mathbf{C}^{n-1}$ for generic projections $p: \mathbf{C}^{n} \rightarrow \mathbf{C}^{n-1}$. Hence all tangent vector fields near $\pi(x)$ lift to vector fields near a smooth point $x$; therefore, by Zariski's lemma [23, Corollary to Th. 30.1], all smooth points on $X$ can be regarded as equisingular, since they have isomorphic analytic neighbourhoods. Of course, this must be so since $\pi$ is étale at $x$, so $\mathcal{O}_{x}$ is analytically isomorphic to the ring or formal power series (or the analytic localisation of $\mathcal{O}_{x}$ is isomorphic to the ring of convergent power series in $n-1$ variables). The next stratum consists of points $x$ where the discriminant of $\pi_{x}$ is non-empty but smooth for generic $p$, so that, at $\pi(x) \in D_{\pi}$, the stalk $T_{D_{\pi}, \pi(x)}$ of tangent vector fields on $D_{\pi}$ near $\pi(x)$ has a basis of non-vanishing tangent vector fields, which extend to non-vanishing vector fields near $\pi(x)$ in $\mathbf{C}^{n-1}$ tangent to $D_{\pi}$; hence they are liftable. Again by Zariski's lemma, points with smooth discriminants (and generic $p$ ) can be regarded as equisingular, since they have isomorphic analytic neighbourhoods. If the generic discriminants $D_{\pi}$ are not smooth, by induction in the dimension $n$ the equisingularity stratification of $D_{\pi}$ is defined, which can be pulled back to get a stratification of $X$. For a more complete discussion of Zariski's equisingularity stratification, see [21,34]. Another situation where a good description of $T_{Y / S}^{\pi}$ is useful is for describing direct images of $D$-modules (see [5]) with respect to a morphism $\pi$ of complex algebraic manifolds, say the zeroth direct image of the structure sheaf $R^{0} \pi_{+}\left(\mathcal{O}_{X}\right)=\pi_{*}\left(\omega_{X / Y} \otimes_{\mathcal{D}_{X}} \pi^{*}\left(\mathcal{D}_{Y}\right)\right.$ ) (in the complement of $D_{\pi}$ it is a Gauss-Manin connection), where $\mathcal{D}_{X}$ and $\mathcal{D}_{Y}$ are the rings of differential operators on $X$ and $Y$, and $\omega_{X / Y}$ is the relative canoncial bundle. Then the characteristic ideal of $R^{0} \pi_{+}\left(\mathcal{O}_{X}\right)$ contains the involutive ideal $J=J\left(T_{Y}^{\pi}\right)$ generated by the symbols of $T_{Y}^{\pi}$ in the graded ring gr $\mathcal{D}_{Y}=\oplus \mathcal{D}_{Y}^{n} / \mathcal{D}_{Y}^{n-1}$, hence the singular support satisfies

$$
\operatorname{SS} R^{0} \pi_{+}\left(\mathcal{O}_{X}\right) \subset J^{-1}(0) \subset T_{Y}^{*} .
$$

Standard notions. Let $A$ and $R$ be local rings $\left(A, \mathfrak{m}_{A}, k_{A}\right)$ and $\left(R, \mathfrak{m}_{R}, k_{R}\right)$, where $k_{A}, k_{R}$ are the residue fields, and $\pi: A \rightarrow R$ a homomorphism of rings. Then $\pi$ is local if $\pi\left(\mathfrak{m}_{A}\right) \subset \mathfrak{m}_{R}, R$ dominates $A$ if $\pi^{-1}\left(\mathfrak{m}_{R}\right)=\mathfrak{m}_{A}$, and $\pi$ is (separably) 
algebraic if the extension of fraction fields $K(R) / K(A)$ is (separably) algebraic, residually algebraic/finite/separable if $k_{R} / k_{A}$ is algebraic/finite/separable; in particular, $R / A$ is residually finite if it is quasi-finite, while the converse holds if $R \mathfrak{m}_{A}$ is an ideal of definition of $R$. We refer to [13,23, for the basic results about formally smooth/unramified morphisms; the discrete topology is intended if no other topology is mentioned. A morphism of schemes $\pi: X \rightarrow Y$ is dominant if the morphism $\pi^{-1}\left(\mathcal{O}_{Y}\right) \rightarrow \mathcal{O}_{X}$ is injective; $\pi$ is generically (separably) algebraic if $\pi$ is dominant, $X$ and $Y$ are reduced, and for points $x \in X$ that map to a generic point $\xi \in Y$ the corresponding residue field extension $k_{x} / k_{\xi}$ is (separably) algebraic, and in particular formally étale. As common practice, by an $\mathcal{O}_{X}$-module we mean a sheaf of modules, and by writing $m \in M$ for a sheaf $M$ we mean that $m$ is a section of $M$ over a suitably defined open set. By a Lie algebroid on $X / S$ is intended an $\mathcal{O}_{X}$-module $\mathfrak{g}_{X / S}$ which moreover is a Lie algebra, provided with a homomorphism (as Lie algebras and $\mathcal{O}_{X}$-modules) to the tangent sheaf $\alpha: \mathfrak{g}_{X / S} \rightarrow T_{X / S}$, with the obvious compatibility relations $[\delta, f \eta]=\alpha(\delta)(f) \eta+f[\delta, \eta], \delta, \eta \in \mathfrak{g}_{X / S}, f \in \mathcal{O}_{X}$ (see e.g. [19]). We shall only have occasions to study Lie algebroids where $\alpha$ is injective. For example, the sub-sheaf of liftable derivations $T_{Y / S}^{\pi}$ is a sub-Lie algebroid of $T_{Y / S}$; other sub-Lie algebroids arise from (fractional) ideals $I$ of $\mathcal{O}_{Y}$, as the subsheaf $T_{Y / S}(I)$ of $T_{Y / S}$ of derivations $\partial$ that preserve $I, \partial(I) \subset I$.

\section{TANGENT MORPHISMS, LOGARITHMIC DERIVATIONS AND RAMIFICATION}

The purpose of the material in Sections 1.4 and 1.5, which is not the minimal necessary to prove our main results, is to provide a useful collection of basic algebraicity/finiteness facts and some ramification theory, with extensions of wellknown results, and relate these classical notions to our lifting problem. For instance, we recall concisely the relation between the property that a morphism $A \rightarrow R$ be finite and quasi-finite, respectively (Prop. 1.4.1) (a formulation of Zariski's main theorem), and the relation between the property that $K(R) / K(A)$ and $k_{R} / k_{A}$ be algebraic, respectively (Prop. 1.4.3). We say a homomorphism of local Noetherian rings $A \rightarrow R$ is ramified if the cotangent mapping $k_{R} \otimes \mathfrak{m}_{A} / \mathfrak{m}_{A}^{2} \rightarrow \mathfrak{m}_{R} / \mathfrak{m}_{R}^{2}$ is not injective. This notion of ramification is adequate for the study of derivations, and in the important case when $R$ is a discrete valuation ring this is close to, but stronger than, formally unramified; we describe what ramification means for the liftable derivations (Prop. 1.5.1). Formally unramified/étale morphisms are described in some detail, complementing the literature: Proposition 1.5.2 is a characterisation of separably algebraic field extensions in terms of vanishing differentials, and Theorem 1.5.4 characterises formally unramified morphisms of Notherian rings $R / A$ as morphisms such that $k_{R} / k_{A}$ is separably algebraic and $R \mathfrak{m}_{A}=\mathfrak{m}_{R}$, removing a finiteness assumption in Auslander and Buchsbaum's proof of the assertion [3]. Lemma 1.5.8 states that separably algebraic field extensions $k^{\prime} / k$ extend to $\mathfrak{m}$-étale extensions of complete $p$-rings (although not étale in the discrete topology); this is needed in the proof of Theorem 2.2.1 to handle unequal characteristics.

1.1. The tangent morphism. A morphism of $S$-schemes $\pi: X / S \rightarrow Y / S$ has its canonical exact sequence of differentials

$$
\pi^{*}\left(\Omega_{Y / S}\right) \rightarrow \Omega_{X / S} \rightarrow \Omega_{X / Y} \rightarrow 0
$$


which induces a homomorphism of $\mathcal{O}_{Y}$-modules, the tangent morphism of $\pi$,

$$
d \pi: T_{X / S}=\operatorname{Hom}_{\mathcal{O}_{X}}\left(\Omega_{X / S}, \mathcal{O}_{X}\right) \rightarrow T_{X / S \rightarrow Y / S}
$$

where the $\mathcal{O}_{X}$-module of 'derivations from $\mathcal{O}_{Y}$ to $\mathcal{O}_{X}$ ' is

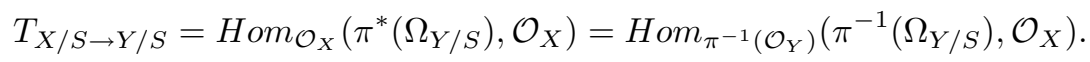

There are canonical homomorphisms:

$$
\begin{gathered}
\psi_{0}: \pi^{-1}\left(T_{Y / S}\right) \rightarrow T_{X / S \rightarrow Y / S}, \\
\psi: \pi^{*}\left(T_{Y / S}\right) \rightarrow T_{X / S \rightarrow Y / S} .
\end{gathered}
$$

If $\pi$ is dominant, then $\psi_{0}$ is injective, but $\psi$ need be neither surjective nor injective when $Y / S$ is non-smooth.

Example 1.1.1. (i) Let $X=\operatorname{Spec} k[t], Y=\operatorname{Spec} k\left[t^{2}, t^{3}\right]$, and $S=\operatorname{Spec} k$. Then $T_{X / k}=\mathcal{O}_{X} \partial_{t}$ and $T_{Y / k}=\mathcal{O}_{Y} t \nabla+\mathcal{O}_{Y} \nabla$, where $\nabla=t \partial_{t}$. Let $\pi$ be the morphism of schemes induced by the inclusion of rings. Then $\partial_{t} \in T_{X / S}$, and $t \otimes \nabla-1 \otimes t \nabla \in$ $\pi^{*}\left(T_{Y}\right)$ is non-zero, while $\psi(t \otimes \nabla-1 \otimes t \nabla)=0$. Also $d \pi\left(\partial_{t}\right)$ is the non-zero section of $T_{X / S \rightarrow Y / S}$ that is induced by the derivation $\partial_{t}: k\left[t^{2}, t^{3}\right] \rightarrow k[t]$; hence $d \pi\left(\partial_{t}\right) \notin \operatorname{Im}(\psi)=\mathcal{O}_{Y} \nabla$. So $\psi$ is neither injective nor surjective. Here $d \pi$ is injective.

(ii) (Immersion of singular locus) Assume that $X / k$ is a variety of characteristic 0 , with singular locus $Z$; let $I_{Z}$ be the ideal of $Z$. Let $\pi: Z \rightarrow X$ be the inclusion of the locus of non-smooth points and $T_{X / k}\left(\mathcal{O}_{X}, I_{Z}\right)$ the subsheaf of $T_{X / k}$ consisting of derivations that send $\mathcal{O}_{X}$ to $I_{Z}$. We have an exact sequence

$$
0 \rightarrow \pi^{*}\left(T_{X / k}\left(\mathcal{O}_{X}, I_{Z}\right)\right) \rightarrow \pi^{*}\left(T_{X / k}\right) \stackrel{\psi}{\rightarrow} T_{Z / k \rightarrow X / k} \rightarrow \mathcal{F}_{Z / X} \rightarrow 0,
$$

where the cokernel $\mathcal{F}_{Z / X}$ of $\psi$ is a subsheaf of $\operatorname{Ext}_{\mathcal{O}_{X}}^{1}\left(\Omega_{X / k}, I_{Z}\right)$. The support of $\mathcal{F}_{Z / X}$ consists of points where $X$ is not equisingular, in the strong sense that there exist tangent vector fields on $Z$ that cannot be extended to tangent vector fields on $X$. Since $T_{X / k}=T_{X / k}\left(I_{Z}\right)$ (see e.g. [19]) there exists a natural homomorphism can $: \pi^{*}\left(T_{X / k}\right) \rightarrow T_{Z / k}$, and a commutative diagram:

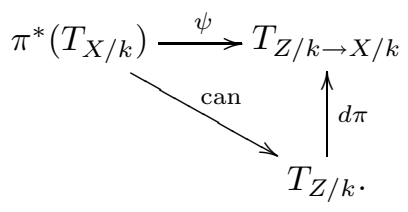

This gives a surjective homomorphism $\mathcal{F}_{Z / X} \rightarrow \mathcal{C}_{Z / X}$. Therefore $d \pi$ is surjective if $\psi$ is surjective.

Proposition 1.1.2. Assume that $Y / S$ is locally of finite type. The homomorphism $\psi$ is an isomorphism in the following cases:

(1) $Y / S$ is smooth.

(2) $\pi$ is flat.

Proof. The map $\psi$ is an isomorphism if it induces an isomorphism of stalks, and since $\Omega_{Y / S}$ is coherent and $\Omega_{Y / S, y} \cong \Omega_{\mathcal{O}_{Y, y} / \mathcal{O}_{S, s}}$ (if $U \subset Y$ is affine and $y \in U$, then the canonical mapping $\mathcal{O}_{Y}(U) \rightarrow \mathcal{O}_{Y, y}$ is étale; then apply the first fundamental exact sequence for differentials)

$$
\operatorname{Hom}_{\mathcal{O}_{Y}}\left(\Omega_{Y / S}, \mathcal{O}_{Y}\right)_{y}=\operatorname{Hom}_{\mathcal{O}_{Y, y}}\left(\Omega_{\mathcal{O}_{Y, y} / \mathcal{O}_{S, s}}, \mathcal{O}_{Y, y}\right) \text {, }
$$


where $s$ is the image of $y$ in $S$, the condition is that the canonical morphism

$$
\psi_{x}: \mathcal{O}_{x} \otimes_{\mathcal{O}_{y}} \operatorname{Hom}_{\mathcal{O}_{y}}\left(\Omega_{\mathcal{O}_{y} / \mathcal{O}_{s}}, \mathcal{O}_{y}\right) \rightarrow \operatorname{Hom}_{\mathcal{O}_{x}}\left(\mathcal{O}_{x} \otimes_{\mathcal{O}_{y}} \Omega_{\mathcal{O}_{y} / \mathcal{O}_{s}}, \mathcal{O}_{x}\right)
$$

be an isomorphism, where $\mathcal{O}_{y} \rightarrow \mathcal{O}_{x}$ is the homomorphism of local rings defined by $\pi$ and points $x \in X, y=\pi(x) \in Y$. By $(1), \Omega_{\mathcal{O}_{y} / \mathcal{O}_{s}}$ is free of finite rank over $\mathcal{O}_{y}$ [14, Prop. 17.2.3], implying the assertion. Since $\Omega_{\mathcal{O}_{y} / \mathcal{O}_{s}}$ is of finite presentation, (2) implies the assertion by [23, Th. 7.11].

When $\psi$ is an isomorphism one gets the "ordinary" tangent homomorphism

$$
\psi^{-1} \circ d \pi: T_{X / S} \rightarrow \pi^{*}\left(T_{Y / S}\right) \text {. }
$$

If $\psi$ is not an isomorphism one can take the fibre product to get a restricted tangent sheaf

$$
\begin{aligned}
T_{X / S}^{r}=\pi^{*}\left(T_{Y / S}\right) \times_{T_{X / S \rightarrow Y / S}} T_{X / S} & \\
= & \left\{(\delta, \partial) \in \pi^{*}\left(T_{X / S}\right) \times_{T_{X / S \rightarrow Y / S}} T_{X / S}: \psi(\delta)=d \pi(\partial)\right\},
\end{aligned}
$$

so the projection on the first factor $T_{X / S}^{r} \rightarrow \pi^{*}\left(T_{Y / S}\right)$ can also play the role of 'tangent morphism'. The sheaf $T_{X / S}^{r}$ is an $\mathcal{O}_{Y}$-Lie algebroid (namely the pull-back of the Lie algebroid $\left.T_{Y / S}\right)$ containing the Lie subalgebroid $T_{X / Y}=\operatorname{Ker}(d \pi)$ of relative tangent vector fields and also the $\mathcal{O}_{X}$ Lie algebra $\mathfrak{b}_{X}=\operatorname{Ker}(\psi)$. One gets a commutative diagram of $\mathcal{O}_{X}$-modules:

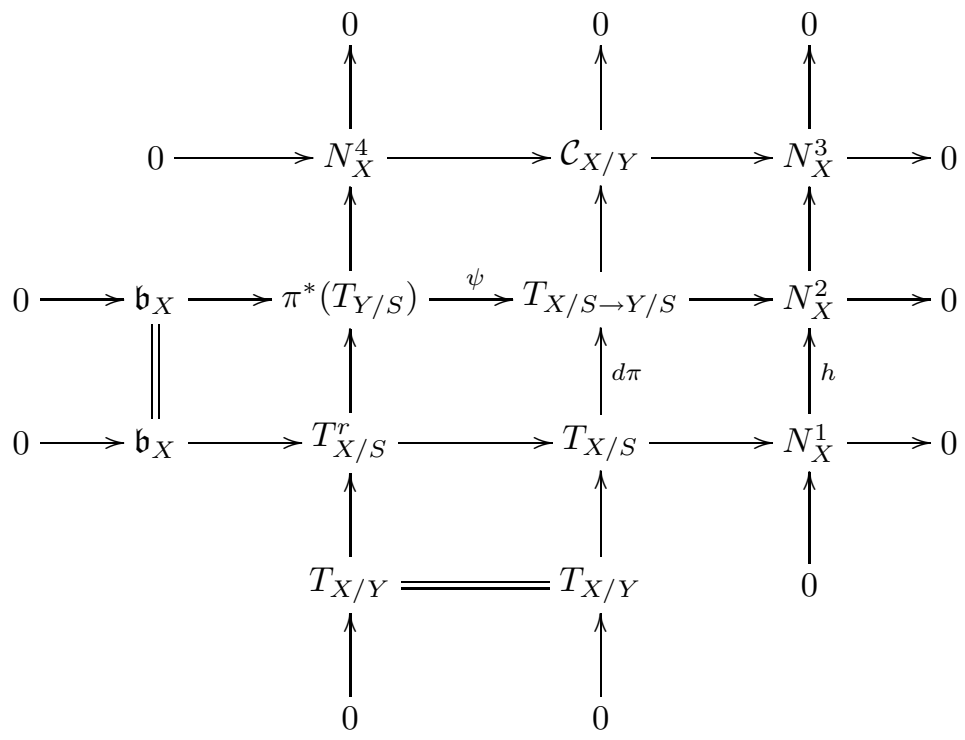

1.2. Submersions and weak submersions. Consider the above vertical sequence containing $d \pi$. The stalk at $x \in X$ of the tangent morphism

$$
d \pi_{x}: T_{X / S, x} \rightarrow T_{X / S \rightarrow Y / S, x}
$$

induces a map of fibres $d \bar{\pi}_{x}: k_{x} \otimes_{\mathcal{O}_{x}} T_{X / S, x} \rightarrow k_{x} \otimes_{\mathcal{O}_{x}} T_{X / S \rightarrow Y / S, x}$; if $T_{X / S \rightarrow Y / S} \cong$ $\pi^{*}\left(T_{X / S}\right)$ (Prop. 1.1.2) one gets the "ordinary" map of tangent spaces $k_{x} \otimes_{\mathcal{O}_{x}}$ $T_{X / S, x} \rightarrow k_{x} \otimes \mathcal{O}_{y} T_{Y / S, y}$. We say that $\pi$ is submersive at $x$ if $d \pi_{x}$ is surjective, i.e. $\mathcal{C}_{X / Y, x}=0$. If $\mathcal{C}_{X / Y, x}$ is of finite type, by Nakayama's lemma, $d \pi_{x}$ is surjective if the map $d \bar{\pi}_{x}$ is surjective. The relation between the critical locus $C_{\pi}=\operatorname{supp} \mathcal{C}_{X / Y}$ and the ramification locus of a morphism is studied in [18. 
There are canonical homomorphisms of $\mathcal{O}_{Y}$-modules

$$
\pi_{*}(d \pi): \pi_{*}\left(T_{X / S}\right) \rightarrow \pi_{*}\left(T_{X / S \rightarrow Y / S}\right) \text { and } \pi_{*}\left(\psi_{0}\right): T_{Y / S} \rightarrow \pi_{*}\left(T_{X / S \rightarrow Y / S}\right),
$$

so one can form the fibre product

$$
\pi_{*}\left(T_{X / S}\right) \times_{\pi_{*}\left(T_{X / S \rightarrow Y / S}\right)} T_{Y / S}=\left\{(\delta, \partial) \in \pi_{*}\left(T_{X / S}\right) \times T_{Y / S}: \pi_{*}(d \pi)(\delta)=i(\partial)\right\} .
$$

Definition 1.2.1. The sub-sheaf $T_{Y / S}^{\pi} \subset T_{Y / S}$ of liftable derivations on $Y$ is the image of the projection on the second factor

$$
T_{Y / S}^{\pi}=\operatorname{Im}\left(\pi_{*}\left(T_{X / S}\right) \times_{\pi_{*}\left(T_{X / S \rightarrow Y / S}\right)} T_{Y / S} \rightarrow T_{Y / S}\right) .
$$

The discriminant module $\mathcal{E}_{X / Y}$ is the cokernel of the inclusion morphism $T_{Y / S}^{\pi} \hookrightarrow$ $T_{Y / S}$ and $D_{\pi}^{\text {disc }}=\operatorname{supp} \mathcal{E}_{X / Y}$ the weak discriminant set of $\pi$. Say that $\pi$ is weakly submersive if $\mathcal{E}_{X / Y}=0$. There are adjoint homomorphisms

$$
\begin{aligned}
\phi_{0} & : \pi^{-1}\left(\mathcal{E}_{X / Y}\right) \rightarrow \mathcal{C}_{X / Y}, \\
\phi & : \mathcal{E}_{X / Y} \rightarrow \pi_{*}\left(\mathcal{C}_{X / Y}\right) .
\end{aligned}
$$

Proposition 1.2.2. (1) If $T_{X / Y}=0$ and $\psi_{0}$ (see (1.2)) is injective, then $\phi_{0}$ is injective.

(2) If $R^{1} \pi_{*}\left(T_{X / Y}\right)=0$ and the composed morphism

$$
T_{Y / S} \rightarrow \pi_{*} \pi^{-1}\left(T_{Y / S}\right) \stackrel{\pi_{*}\left(\psi_{0}\right)}{\longrightarrow} \pi_{*}\left(T_{X / S \rightarrow Y / S}\right)
$$

is injective, then $\phi$ is injective.

(3) Assume that $\pi_{*}\left(\mathcal{O}_{X}\right)=\mathcal{O}_{X}$ (e.g. $\pi$ is proper and birational, and $Y$ is normal), and $Y / S$ is smooth. Assume also that $\pi_{*}\left(T_{X / Y}\right)=0, R^{1} \pi_{*}\left(T_{X / Y}\right)=$ 0 (e.g. $\left.T_{X / Y}=0\right)$ and that $\psi($ see (1.3) $)$ is an isomorphism (Prop. 1.1.2). Then $T_{Y / S}^{\pi}=\pi_{*}\left(T_{X / S}\right), \mathcal{E}_{X / Y}=\pi_{*}\left(\mathcal{C}_{X / Y}\right)$, and in particular $D_{\pi}^{\text {disc }}=$ $\pi\left(C_{\pi}\right)$.

If either of the conditions in (1) or (2) holds and $\pi$ is submersive, then $\pi$ is weakly submersive.

Assuming the conditions in (1), which is the situation we shall mostly deal with, then in general when $\psi$ is not an isomorphism we have a strict inclusion $D_{\pi}^{d i s c} \subset \pi\left(C_{\pi}\right)$, so the notion of submersive morphism is stronger than that of weakly submersive morphism; it is in fact straightforward to see that these notions coincide (assuming (1)) if and only if $\psi$ is an isomorphism. In Example 1.2.3 below the morphism $\pi$ is weakly submersive but not submersive; the fact that this normalisation morphism is weakly submersive is not coincidental (Th. 2.3.1).

To describe the sheaf $T_{Y / S}^{\pi}$ we shall use the critical set $C_{\pi}$ and the closure $D_{\pi}$ of $\pi\left(C_{\pi}\right)$, rather than the weak discriminant set $D_{\pi}^{\text {disc }}$ (in case $D_{\pi}^{\text {disc }} \neq \pi\left(C_{\pi}\right)$ ).

Proof. (1): Since $T_{X / Y}=0$ and $\pi^{-1}$ is exact we have a commutative diagram of $\pi^{-1}\left(\mathcal{O}_{Y}\right)$-modules:

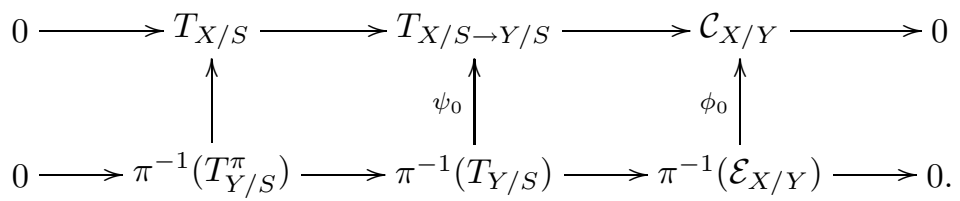

Clearly, if $\psi_{0}$ is injective, then $\phi_{0}$ is injective. 
(2): Letting $\bar{T}_{X / S}$ be the image of $d \pi$ we have a corresponding commutative diagram of $\mathcal{O}_{Y}$-modules:

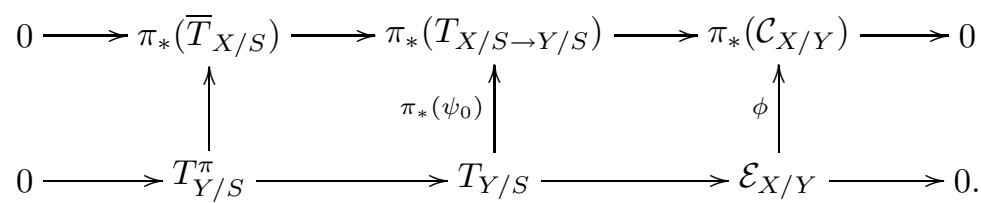

Here the left vertical map is the composition $T_{Y / S}^{\pi} \rightarrow \pi_{*}\left(T_{X / S}\right) \rightarrow \pi_{*}\left(\bar{T}_{X / S}\right)$ where by assumption the latter map is surjective. By the definition of $T_{Y / S}^{\pi}$ the assertion now follows.

(3): The assumption implies $\pi_{*}\left(T_{X / S}\right)=\pi_{*}\left(\bar{T}_{X / S}\right)$, hence by (2) the map $\phi$ is injective, and since $\psi_{0}$ is injective and therefore $\pi_{*}\left(\psi_{0}\right)$ is injective, the left vertical map is injective by the definition of $T_{Y / S}^{\pi}$. Therefore $T_{Y / S}^{\pi}=\pi_{*}\left(T_{X / S}\right)$ and $\mathcal{E}_{X / Y}=\pi_{*}\left(\mathcal{C}_{X / Y}\right)$ follows if the middle vertical map is surjective. Since $\psi$ is an isomorphism the assertion follows if the canonical map $T_{Y / S} \rightarrow \pi_{*} \pi^{*}\left(T_{Y / S}\right)$ is an isomorphism. This follows from the projection formula since $Y / S$ is smooth, so $T_{Y / S}$ is locally free, and since we have $\pi_{*} \pi^{*}\left(\mathcal{O}_{Y}\right)=\mathcal{O}_{Y}$.

The remaining assertion is evident, since if $\phi$ or $\phi_{0}$ are injective, it follows that $\operatorname{supp} \mathcal{E}_{X / Y} \subset \operatorname{supp} \mathcal{C}_{X / Y}$.

Let $k \rightarrow A \stackrel{\pi}{\rightarrow} R$ be homomorphisms of rings, and $T_{A / k}=\operatorname{Hom}_{A}\left(\Omega_{A / k}, A\right)$ and $T_{R / k}$ be the Lie algebroid of $k$-linear derivations of $A$ and $R$, respectively. The tangent morphism $d \pi: T_{R / k} \rightarrow T_{A / k \rightarrow R / k}=H_{o m}\left(\Omega_{A / k}, R\right)=$ $\operatorname{Hom}_{R}\left(R \otimes_{A} \Omega_{A / k}, R\right)$ is given by $d \pi(\partial)(d a)=\partial(\pi(a)), \partial \in T_{R / k}, a \in A$. Let $\psi_{0}: T_{A / k} \rightarrow T_{A / k \rightarrow R / k}$ be the natural morphism, which is injective if $\pi$ is injective. The liftable derivations are $T_{A / k}^{\pi}=\operatorname{Im}(d \pi) \cap \psi\left(T_{A / k}\right)$.

Example 1.2.3. We continue with Example1.1.1 (i). We have $T_{A / k \rightarrow R / k}=R \frac{1}{t} \partial_{t}$ and $\mathcal{C}_{R / A}=T_{A / k \rightarrow R / k} / T_{R / k}=R / \mathfrak{m}_{R}=k$, but $T_{A / k}^{\pi}=T_{A / k}$, so $\mathcal{E}_{R / A}=0$.

Liftable derivations may be included in $\mathfrak{m}_{R}^{N} T_{R}$ for any high $N$. We give a random example that $N$ can be high (in this case $N=9$ ).

Example 1.2.4. Let $A$ be a $\mathbf{Q}$-algebra with normalisation $R=\mathbf{Q}[[t]], A \neq R$. Then $T_{R}^{c o n t}=T_{R / \mathbf{Q}}^{c o n t}=R \partial_{t}$ (continuous derivations; alternatively, work instead with the localisation $\left.R=\mathbf{Q}[t]_{0}\right)$. There exists a smallest integer $c$ such that $\left(t^{c}\right) \in A$. Put $R_{c}=R /\left(t^{c}\right)$ and $A_{c}=A /\left(t^{c}\right)$, so $A_{c} \subset R_{c}$ is an inclusion of finite-dimensional Q-algebras. A derivation of $R$ that preserves $A$ is of the form $\nabla=f t \partial_{t}, f \in R$, so $\nabla$ also induces a derivation of $A_{c}$ and $R_{c}$. If $\bar{a}_{1}, \ldots, \bar{a}_{l}$ are generators of $A_{c}$, where $\bar{a}_{i} \in R_{c}$ is the projection of $a_{i} \in R$, the condition on $\bar{f}$ is $\bar{f} \bar{a}_{i}^{\prime} \in A_{c}, i=1,2,3$, where $\bar{a}_{i}^{\prime}=\overline{t \partial_{t}\left(a_{i}\right)}$. These equations may have only the trivial solution $f \in\left(t^{c}\right)$. A concrete example is $A=\mathbf{Q}\left[\left[a_{1}, a_{2}, a_{3}, t^{9}, t^{10}, \ldots\right]\right], a_{1}=t^{4}+t^{7}, a_{2}=t^{5}+t^{8}, a_{3}=t^{6}$. We indicate that $T_{A}^{\pi} \subset \mathfrak{m}_{R}^{9} T_{R}$. The ramification index is 4 and $c=9$, so working in $R_{9}$ we see that $\overline{1}, \bar{a}_{1}, \bar{a}_{2}, \bar{a}_{3}$ forms a $\mathbf{Q}$-basis for the 4-dimensional subspace $A_{9}$ $\left(\bar{a}_{i} \cdot \bar{a}_{j}=0, i \neq j\right)$ of the 9 -dimensional space $R_{9}$. To solve $\bar{f} \bar{a}_{i}^{\prime} \in A_{9}$ it suffices to make the ansatz $\bar{f}=c_{0}+c_{1} t+c_{2} t^{2}+c_{3} t^{3}+c_{4} t^{4} \in R_{9}, c_{i} \in \mathbf{Q}$, so we have the equations $\left(c_{0}+c_{1} t+c_{2} t^{2}+c_{3} t^{3}+c_{4} t^{4}\right) a_{i}^{\prime} \equiv \alpha_{i 1} a_{1}+\alpha_{i 2} a_{2}+\alpha_{i 3} a_{3} \bmod \left(t^{9}\right), i=1,2,3$. Identifying coordinates in a basis of the 9 -dimensional space $R_{9}$ gives 5 equations for each $i$, so there are 15 equations. There are 5 parameters in $\bar{f}$ and the $\alpha_{i j}$ give 
another 9 parameters, giving 14 parameters. A straightforward computation shows that the only solution is $\bar{f}=0$. Note that the vectors $\left(c_{0}+c_{1} t+\cdots+c_{4} t^{4}\right) a_{i}^{\prime}$ span a 5 -dimensional subspace while the vectors $\alpha_{i 1} a_{1}+\alpha_{i 2} a_{2}+\alpha_{i 3} a_{3}$ span a 3 -dimensional subspace of $R_{9}$, so we should expect that their intersection is 0 .

1.3. Logarithmic derivations. Let $k \rightarrow A \stackrel{\pi}{\rightarrow} R$ be inclusions of rings, where we regard $A$ and $R$ as algebras over $k$; e.g. $k=\mathbf{Z}$, the ring of integers, and $R$ is a discrete valuation ring. Assume that the extension of fraction fields $K(R) / K(A)$ is separably algebraic, and therefore that the $A$-module of derivations $T_{A / k}$ can be regarded as an $A$-Lie subalgebroid of $T_{K(R) / k}, T_{A / k} \subset T_{K(R) / k}$; put $T_{A / k}^{\pi}=$ $T_{A / k} \cap T_{R / k}$.

Definition 1.3.1. Denote by $T_{A / k}\left(\log ^{\pi} \mathfrak{m}_{A}\right)$ the $A$-submodule of $T_{A / k}$ of derivations $\partial$ such that $\partial(\phi) \in R \phi$ when $\phi \in \mathfrak{m}_{A}$, and denote by $T_{A / k}\left(\mathfrak{m}_{A}\right)$ the $A$-module of derivations such that $\partial\left(\mathfrak{m}_{A}\right) \subset \mathfrak{m}_{A}$.

The Lie algebroid $T_{A / k}\left(\log ^{\pi} \mathfrak{m}_{A}\right)\left(=T_{A / k}\left(\log ^{\pi}\left\{x_{1}, \ldots, x_{r}\right\}\right)\right.$ for a basis $\left\{x_{1}, \ldots\right.$, $\left.x_{r}\right\}$ of $\left.\mathfrak{m}_{A}\right)$ is a sub-Lie algebroid of the Lie algebroid $T_{A / k}\left(\mathfrak{m}_{A}\right)$; elements in the former module are $\pi$-logarithmic and elements in the latter are logarithmic derivations.

Lemma 1.3.2. Let $L \subset K(A)$ be a subset. Denote by $T_{A / k}\left(\log ^{\pi} L\right)$ the $A$-submodule of $T_{A / k}$ of derivations $\partial$ such that $\partial(\phi) \in R \phi$ when $\phi \in L$. Then

$$
T_{A / k}\left(\log ^{\pi} L\right) \subset T_{A / k}\left(\log ^{\pi} \mathfrak{m}_{A}\right) \subset T_{A / k}\left(\mathfrak{m}_{A}\right),
$$

where the latter is an inclusion of Lie algebroids. If $L$ is either a non-zero $A$ submodule of $K(A)$ or contains a basis of $\mathfrak{m}_{A}$, then $T_{A / k}\left(\log ^{\pi} L\right)=T_{A / k}\left(\log ^{\pi} \mathfrak{m}_{A}\right)$.

$\underline{\text { Note: }}$ If $P$ is an $\mathfrak{m}_{A}$-primary ideal, the Lie algebroids $T_{A / k}(P)$ and $T_{A / k}\left(\mathfrak{m}_{A}\right)$ are in general not included in one another, but if Char $A=0$, then $T_{A / k}(P) \subset$ $T_{A / k}\left(\mathfrak{m}_{A}\right)$ (see e.g. [19]). Note also that for general subsets $L \subset K(A)$ we have that $T_{A / k}(L)=\left\{\partial \in T_{A / k} \mid \partial(\phi) \in L\right.$ when $\left.\phi \in L\right\}$ is a Lie subalgebroid of $T_{A / k}$, but the $A$-module $T_{A / k}\left(\log ^{\pi} L\right)$ need not be a Lie algebra. Moreover, in general, $T_{A / k}\left(\log ^{\pi} L\right) \not \subset T_{A / k}(L)$, also when $L$ is an ideal of $A$.

The global version of the above definition is as follows. Let $\pi: X \rightarrow Y$ be a dominant generically separably algebraic morphism of schemes, where $X$ is a Krull scheme, i.e. locally the spectrum of a Krull ring. A Krull ring is an integral domain formed as an intersection $A=\bigcap_{\lambda} R_{\lambda}$ of discrete valuation rings $R_{\lambda}$ in its fraction field $K(A)$ such that every non-zero element in $K(A)$ is invertible in all but finitely many $R_{\lambda} 11$ a locally Nœetherian scheme is Krull if and only if it is integral and integrally closed in its fraction field. Polynomial rings in infinitely many variables over a field are non-Nœetherian Krull rings. See [6, Ch. VII, $§ 1.3 ;$ 9; 24, Ch. V] for a treatment of Krull rings.

For a closed subset $D \subset Y$ the sheaf of logarithmic derivations along $D$ is $T_{Y / S}\left(I_{D}\right)=T_{Y / S}(\log D)=\left\{\partial \in T_{Y / S} \mid \partial\left(I_{D}\right) \subset I_{D}\right\}$, where $I_{D}$ is the (reduced) ideal of $D$. Let $C=\bigcup C_{i}$ be a union of irreducible closed subsets of pure codimension 1 in $X$. Let $\mathcal{O}_{X, c_{i}}$ be the local ring at the generic point $c_{i}$ of $C_{i}$, and $\nu_{i}$ the associated normalised discrete valuation. The presheaf of $\pi$-logarithmic derivations

${ }^{1}$ We will need only the first condition for our main results. 
along $C$ is

$$
\begin{aligned}
T_{Y / S}\left(\log ^{\pi} C\right)=\left\{\partial \in T_{Y / S} \mid \nu_{c_{i}}\left(\pi_{c_{i}}(\partial(f))\right) \geq\right. & \nu_{c_{i}}\left(\pi_{c_{i}}(f)\right) \\
& \text { when } \left.f \in \mathcal{O}_{Y, \pi\left(c_{i}\right)}, i=1, \ldots, r\right\},
\end{aligned}
$$

where $\pi_{c}$ denotes the homomorphism of local rings $\mathcal{O}_{Y, \pi(c)} \rightarrow \mathcal{O}_{X, c}$. It is easy to see that $T_{Y / S}\left(\log ^{\pi} C\right)$ actually is a sheaf, and to see that a section $\partial$ of $T_{Y / S}$ is $\pi$-logarithmic, it suffices to check that its germ $\partial_{\pi\left(c_{i}\right)} \in T_{\mathcal{O}_{Y, \pi\left(c_{i}\right)} / \mathcal{O}_{S, s_{i}}}\left(\log ^{\pi} \mathfrak{m}_{\pi\left(c_{i}\right)}\right)$, where $s_{i} \in S$ is the image of $c_{i} \in Y$. Put $D=\pi(C)$ and let $I_{D}$ be any defining ideal of the closure of $D$, and put $T_{Y / S}\left(\log ^{\pi} I_{D}\right)=\left\{\partial \in T_{Y / S} \mid \nu_{c_{i}}(\partial(f)) \geq\right.$ $\nu_{i}(f)$ for all $\left.\phi \in I, i=1, \ldots, r\right\}$. The notation is incomplete since this sheaf also depends on the choice of $C$ (different $C$ can give the same $D$ ), but we choose not to burden the notation; if $C=\pi^{-1}(D)$, there is no ambiguity and in practice $C$ will always be the critical set $C_{\pi}$ of the morphism $\pi$. To determine if $\partial \in T_{Y / S}$ belongs to $T_{Y / S}\left(\log ^{\pi} I_{D}\right)$ it suffices to check the condition for a set of local generators of $I_{D}$

Lemma 1.3.3. (1) The sheaf $T_{Y / S}\left(\log ^{\pi} I_{D}\right)$ is independent of the choice of the defining ideal $I_{D}$ of the closure of D; more precisely,

$$
T_{Y / S}\left(\log ^{\pi} I_{D}\right)=T_{Y / S}\left(\log ^{\pi} C\right) .
$$

(2) If $I_{D}$ is a defining ideal of $D$, then

$$
T_{Y / S}\left(\log ^{\pi} C\right) \subset T_{Y / S}\left(\sqrt{I}_{D}\right) ;
$$

in particular $T_{Y / S}\left(\log ^{\pi} C\right) \subset T_{Y / S}(\log D)$.

In view of (1) it is reasonable to write

$$
T_{Y / S}\left(\log ^{\pi} D\right)=T_{Y / S}\left(\log ^{\pi} I_{D}\right)=T_{Y / S}\left(\log ^{\pi} C\right),
$$

where $I_{D}$ is an arbitrary defining ideal of the closure of $D$.

Proof. (1): Clearly, $T_{Y / S}\left(\log ^{\pi} C\right) \subset T_{Y / S}\left(\log ^{\pi} I_{D}\right)$, so assuming $\partial$ is a section of $T_{Y / S}\left(\log ^{\pi} I_{D}\right)$, regular at $\pi\left(c_{i}\right)$, and $f \in \mathcal{O}_{Y, \pi\left(c_{i}\right)}$, we have to check that $\nu_{c_{i}}(\partial(f)) \geq$ $\nu_{c_{i}}(f)$. If $f \notin \mathfrak{m}_{\pi\left(c_{i}\right)}$ we have $\nu_{c_{i}}(f)=0$ and the assertion is obvious. If $f \in$ $\mathfrak{m}_{\pi\left(c_{i}\right)}$, then $f^{n} \in I_{\pi\left(c_{i}\right)}$ for sufficiently high $n$; hence $\nu_{c_{i}}\left(\partial\left(f^{n}\right)\right) \geq \nu_{c_{i}}\left(f^{n}\right)$, i.e. $\nu_{c_{i}}\left(n \partial(f) f^{n-1}\right)=\nu_{c_{i}}(\partial(f))+(n-1) \nu_{c_{i}}(\partial(f))=n \nu_{c_{i}}(\partial(f)) \geq n \nu_{c_{i}}(f)$, so $\nu_{c_{i}}(\partial(f))$ $\geq \nu_{c_{i}}(f)$.

(2): In the light of (1) it suffices to prove that a section $\partial$ of $T_{Y / S}\left(\log ^{\pi} I_{D}\right)$ preserves the minimal associated primes of $I_{D}$ for any defining ideal of $D$. A minimal associated prime of $I_{D}$ is of the form $\pi\left(c_{i}\right)$ for some generic point $c_{i}$ of $C$. Therefore $\partial \in T_{Y / S, \pi\left(c_{i}\right)}\left(\log ^{\pi} \mathfrak{m}_{\pi\left(c_{i}\right)}\right) \subset T_{Y / S, \pi\left(c_{i}\right)}\left(\mathfrak{m}_{\pi\left(c_{i}\right)}\right)$ (Lem. 1.3.2) .

\subsection{Quasi-finite and algebraic morphisms.}

Proposition 1.4.1. Let $\pi: A \rightarrow R$ be an injective homomorphism of local integral rings, where $A$ is Notherian. Assume that at least one of the following conditions holds:

(i) A is complete.

(ii) the A-algebra $R$ is of finite type.

(iii) $R$ is integrally closed in $K(R), K(R)$ is finite over $K(A)$, and the integral closure of $A$ in $K(A)$ is finite. 
Then the following are equivalent:

(1) $\operatorname{dim}_{k_{A}} k_{A} \otimes_{A} R<\infty$ ( $R$ is quasi-finite over $\left.A\right)$.

(2) $R$ is finite over $A$.

See 23] for a discussion of the problem with the property that the normalisation be finite over $A$. It suffices that the completion of $A$ be reduced, and if $R$ is a discrete valuation ring, this is also necessary.

Remark 1.4.2. Recall the situation when $A$ is a discrete valuation ring and $K(R) / K(A)$ is finite. Then $k_{R} / k_{A}$ is finite, and the ramification index of $A \rightarrow R$ is the integer $e$ such that $R \mathfrak{m}_{A}=\mathfrak{m}_{R}^{e}$; it is also the index of the value group of $A$ in the value group of $R$. If $A$ moreover is complete, then $[K(R): K(A)]=e\left[k_{R}: k_{A}\right]$.

Proof. We have only to prove $(1) \Rightarrow(2)$. If (i) holds, see [12, Ch. 0, Cor. 7.4.3]. If (ii) holds, this is Zariski's main theorem; see [15, Ch. III, Cor. 4.4.6]. If (iii) holds, it follows that the integral closure $A^{*}$ of $A$ in $K(R)$ is finite over $A[6$, Ch. $\mathrm{V}, \S 1.6$, Prop. 18] and since $R$ is integrally closed, $A^{*} \subset R$. Now $R$ is a union of local quasi-finite $A^{*}$-algebras $F$ of finite type; $K\left(A^{*}\right)=K(R)$ since $K(R) / K(A)$ is algebraic. Therefore $K\left(A^{*}\right)=K(F)$, so by Zariski's main theorem, $F=A^{*}$ [15, Ch. III, Cor. 4.4.8]; hence $R=A^{*}$.

Proposition 1.4.3. Let $\pi: A \rightarrow R$ be a local injective homomorphism of Notherian integral rings. Consider the conditions:

(1) $K(R) / K(A)$ is algebraic.

(2) $k_{R} / k_{A}$ is algebraic.

Then:

(a) If (1) and (2) hold, and $A$ is universally catenary (e.g. formally equidimensional), then $\operatorname{dim} A=\operatorname{dim} R$.

(b) If $R$ is a valuation ring and $A=K(A) \cap R$, then (1) $\Rightarrow$ (2).

(c) If $F \mathfrak{m}_{A}$ is $\mathfrak{m}_{F}$-primary for each local intermediate A-algebra $\left(F, \mathfrak{m}_{F}\right)$ of finite type, $A \subset F \subset R$, then (2) $\Rightarrow(1)$.

Assuming (2), the assumption in (c) is the same as $F / A$ is quasi-finite. Note that if $R / A$ is of finite type and $F$ is a local $A$-algebra as in (c) then if $R / A$ is quasi-finite, it follows that $F / A$ is quasi-finite. To see this, apply $k_{A} \otimes_{A}$. to the exact sequence of $A$-modules $0 \rightarrow F \rightarrow R \rightarrow F / R \rightarrow 0$, noting that $\operatorname{dim}_{k_{A}} \operatorname{Tor}_{1}^{A}\left(k_{A}, R / F\right)<\infty$ when $R$ is of finite type, and by assumption, $\operatorname{dim}_{k_{A}} k_{A} \otimes_{A} R<\infty$. If $R / A$ is not of finite type this does not follow (example: $A=k[x]_{0} \subset R=k[[x]]$ ).

Proof. (a) An algebraic field extension $K(R) / K(A)$ is a union of algebraic extensions $K(F) / K(A)$ where $A \subset F \subset R$ and $F$ is of finite type over $A$. Since $R$ is Notherian, $\operatorname{dim} R=\sup _{A \subset F \subset R} \operatorname{dim} F$; hence it suffices to see that $\operatorname{dim} F=\operatorname{dim} A$. Since $F / A$ is of finite type, we conclude from Ratliff's dimension equality (see [23. Th. 15.6]), since $A$ is Notherian and universally catenary, that (1) - (2) imply $\operatorname{dim} A=\operatorname{dim} R$.

(b) See [6, Ch. VI, $\S 8$, Prop. 1].

(c) By assumption, $R$ is a union of local $A$-algebras $F$ of finite type such that $F \mathfrak{m}_{A}$ is $\mathfrak{m}_{F}$-primary; hence by (2) $F \otimes_{A} k_{A}$ is finite over $k_{A}$. Hence by Zariski's main theorem (Prop. 1.4.1) $F$ is finite over $A$. Since $K(R)$ is a union of the fields $K(F)$ it follows that $K(R)$ is algebraic over $K(A)$. 
1.5. Ramification. We collect some facts about ramifications of injective homomorphisms of local rings $k \rightarrow A \rightarrow R$. The cotangent mapping is denoted

$$
\phi: k_{R} \otimes_{k_{A}} \mathfrak{m}_{A} / \mathfrak{m}_{A}^{2} \rightarrow \mathfrak{m}_{R} / \mathfrak{m}_{R}^{2}
$$

A field extension $k \subset l$ has a finite differential basis if $\operatorname{dim}_{l} \Omega_{l / k}<\infty$, which is equivalent to tr. $\operatorname{deg} l / k<\infty$ when Char $k=0$ and equivalent to $l / k$ having a finite $p$-basis when Char $k=p$ (see [23, $\S 26]$ ). We say that an extension $k \subset l$, where $k$ is a subring of the field $l$, has a finite differential basis if $l$ has a finite differential basis over the fraction field of $k$.

Proposition 1.5.1. Let $\left(A, \mathfrak{m}_{A}, k_{A}\right)$ be a local Noetherian subring of a discrete valuation ring $\left(R, \mathfrak{m}_{R}, k_{R}\right)$, where $\mathfrak{m}_{A} \neq 0$. Let $k \subset A$ be a subring such that the extensions $k_{R} / k$ and $k_{A} / k$ are separable with finite differential bases. Consider the following conditions:

(1) The map $\phi$ is injective.

(2) The ring $\left(A, \mathfrak{m}_{A}, k_{A}\right)$ is a discrete valuation ring and $T_{A / k}=T_{A / k}^{\pi}$.

(3) The tangent morphism $d \pi: T_{R / k} \rightarrow T_{A / k \rightarrow R / k}$ is surjective.

(4) The map $\phi$ is surjective.

Then $(1) \Leftrightarrow(2),(1-2) \Rightarrow(3)$ and $(1-2) \Rightarrow(4)$. If $A / k$ is smooth, then $(3) \Rightarrow$ $(1-2)$. If $A$ is a discrete valuation ring, then $(4) \Rightarrow(1-2)$.

Proof. (1) $\Leftrightarrow(2)$ : Since $A$ is a Notherian integral domain, we have by (1), $1 \leq$ $\operatorname{dim} A \leq \operatorname{dim}_{k_{A}} \mathfrak{m}_{A} / \mathfrak{m}_{A}^{2}$. Hence (1) implies that $\left(A, \mathfrak{m}_{A}, k_{A}\right)$ is a discrete valuation ring; thus in either case we have an inclusion of discrete valuation rings $A \subset R$. Since $\operatorname{dim}_{k_{R}} \Omega_{k_{R} / k}<\infty$ and $\operatorname{dim}_{k_{A}} \Omega_{k_{A} / k}<\infty$ it follows from the second fundamental exact sequence of differentials that $\Omega_{R / k}$ and $\Omega_{A / k}$ are free of finite rank. Therefore $T_{R / k}$ and $T_{A / k \rightarrow R / k}=R \otimes_{A} T_{A / k}$ (see proof of Proposition 1.1.2) are free of finite rank over $R$. It follows by Nakayama's lemma that the map $d \pi: T_{R / k} \rightarrow R \otimes T_{A / k}$ is surjective if and only if the tangent map $k_{R} \otimes_{R} T_{R / k} \rightarrow k_{R} \otimes_{R} T_{A / k}$ is surjective; i.e. the map $\left(\mathfrak{m}_{R} / \mathfrak{m}_{R}^{2}\right)^{*} \oplus T_{k_{R} / k} \rightarrow$ $k_{R} \otimes_{k}\left(\mathfrak{m}_{A} / \mathfrak{m}_{A}^{2}\right)^{*} \oplus k_{R} \otimes_{k_{A}} T_{k_{A} / k}$ is surjective. Since $k_{R} / k_{A}$ is separable, the map $T_{k_{R} / k} \rightarrow T_{k_{A} / k \rightarrow k_{R} / k}=k_{R} \otimes_{k_{A}} T_{k_{A} / k}$ is surjective; hence $d \pi$ is surjective if and only if the map $\left(\mathfrak{m}_{R} / \mathfrak{m}_{R}^{2}\right)^{*} \rightarrow k_{R} \otimes_{k_{A}}\left(\mathfrak{m}_{A} / \mathfrak{m}_{A}^{2}\right)^{*}=H_{o m} m_{k_{A}}\left(\mathfrak{m}_{A} / \mathfrak{m}_{A}^{2}, k_{R}\right)$ is surjective, and this map is surjective if and only if its image contains the image of the natural map $\operatorname{Hom}_{k_{A}}\left(\mathfrak{m}_{A} / \mathfrak{m}_{A}, k_{A}\right) \rightarrow \operatorname{Hom}_{k_{A}}\left(\mathfrak{m}_{A} / \mathfrak{m}_{A}, k_{R}\right)$, i.e. $d \pi$ is surjective if and only if $T_{A / k} \subset d \pi\left(T_{R / k}\right)$. Since (1) is equivalent to $\left(\mathfrak{m}_{R} / \mathfrak{m}_{R}^{2}\right)^{*} \rightarrow k_{R} \otimes_{k_{A}}\left(\mathfrak{m}_{A} / \mathfrak{m}_{A}^{2}\right)^{*}$ being surjective, it follows that $(1) \Leftrightarrow(2)$.

$(1-2) \Rightarrow(3)$ : Since $\Omega_{A / k}$ is (free) of finite type, the map $d \pi$ is surjective if and only if the map $\left(\mathfrak{m}_{R} / \mathfrak{m}_{R}^{2}\right)^{*} \oplus T_{k_{R} / k} \rightarrow k_{R} \otimes_{k_{A}}\left(\mathfrak{m}_{A} / \mathfrak{m}_{A}^{2}\right)^{*} \oplus k_{R} \otimes_{k_{A}} T_{k_{A} / k}$ is surjective. The assertion is then clear from the above argument, as is the assertion $(3) \Rightarrow(1-2)$ when $A / k$ is smooth, for then $\Omega_{A / k}$ is free of finite rank since $\operatorname{dim}_{k_{A}} \Omega_{k_{A} / k}<\infty$.

$(1-2) \Rightarrow(4)$ : If $\phi$ is injective, it is also surjective since $\operatorname{dim} \mathfrak{m}_{R} / \mathfrak{m}_{R}^{2}=1$. If $A$ and $R$ are discrete valuation rings, then $\phi$ is injective if and only if it is surjective.

Proposition 1.5.2. The following are equivalent for a field extension $l / k$ :

(1) $l / k$ is formally étale and algebraic.

(2) $l / k$ is separably algebraic.

(3) $\Omega_{f / k}=0$ for each intermediate field $l / f / k$ such that $f / k$ is of finite type.

If $l / k$ is of finite type or $l / k$ is separable, then (3) is equivalent to $\Omega_{l / k}=0$. 
Example 1.5.3. Let $k$ be a field of characteristic $p>0$ and $l=k\left(x^{p^{-\infty}}\right)=$ $k\left(x, x^{p^{-1}}, x^{p^{-2}}, \ldots\right)$. Then $l / k$ is separable and formally étale, but not algebraic (see [23, Ex. 26.8]); moreover $\Omega_{l / k}=0$, and there exist finite intermediate fields $l / f / k$ such that $\Omega_{f / k} \neq 0$.

$(3) \Rightarrow(2)$ is proven in [13, Cor. 21.7.4] when $l / k$ is of finite type, and the argument below is not very different, but it may be convenient to have it presented in this way.

Proof. (1) $\Leftrightarrow(2)$ : see [23, Thms. 25.3, 26.9]. For the remaining parts let $\Omega_{k}$ and $\Omega_{l}$ denote differentials over the prime field and consider the exact sequence $0 \rightarrow \Gamma \rightarrow l \otimes \Omega_{k} \stackrel{h}{\rightarrow} \Omega_{l} \rightarrow \Omega_{l / k} \rightarrow 0 .(2) \Rightarrow(3)$ : Then $l / k$ is formally étale and $h$ is an isomorphism [loc. cit.]. (3) $\Rightarrow(2)$ : If $k \subset f \subset l$ is an intermediate field such that $f / k$ is of finite type, we have $\Omega_{f / k}=0$, and replacing $l$ by $f$ in the above exact sequence, we see that the map $h$ is surjective, and by Cartier's equality $\operatorname{rk}_{f} \Omega_{f / k}=\operatorname{tr} . \operatorname{deg}_{k} f+\operatorname{rk}_{f} \Gamma$ it follows that tr. $\operatorname{deg}_{k} f=0$ and $\operatorname{rk}_{f} \Gamma=0$; hence $f / k$ is algebraic (hence finite) and $h$ is injective, implying that $f / k$ is separable [loc. cit., Th. 26.6]. Since $l$ is a union of such subfields $f$, it follows that $l / k$ is separably algebraic.

Theorem 1.5.4. Let $\left(A, \mathfrak{m}_{A}, k_{A}\right) \subset\left(R, \mathfrak{m}_{R}, k_{R}\right)$ be an inclusion of local Notherian rings. Consider the conditions:

(1) $\Omega_{R / A}=0$.

(2) $k_{R} / k_{A}$ is separably algebraic and $R \mathfrak{m}_{A}=\mathfrak{m}_{R}$.

(3) $k_{R} / k_{A}$ is separably algebraic and the map $\phi$ is surjective.

(4) $k_{R} / k_{A}$ is separably algebraic and the map $\phi$ is injective.

Then $(2) \Leftrightarrow(3)$ and $(2) \Rightarrow(1)$. If the residue field extension $k_{R} / k_{A}$ is finitely generated, then (1) $\Rightarrow(2)$. If $A$ and $R$ are discrete valuation rings, then (2) $\Leftrightarrow$ $(3) \Leftrightarrow(4)$.

Auslander and Buchsbaum 3 initiated the systematic ramification theory of Nœtherian rings. They proved $(1) \Leftrightarrow(2)$ under the assumption that $J=$ $\operatorname{Ker}\left(R \otimes_{A} R \rightarrow R, r_{1} \otimes r_{2} \mapsto r_{1} r_{2}\right)$ is a finitely generated ideal in $R \otimes_{A} R$, and hence that $\Omega_{R / A}$ is $R$-finite. In [14, $\S 17$, Th. 17.4.1] one finds a different proof of the same assertion assuming $R$ is a, possibly non-Notherian, finitely presented $A$-algebra (implying $J$ is finitely generated), but the argument in [loc. cit.] for $(1) \Rightarrow(2)$ is fairly involved. In Theorem 1.5.4 $J$ is not assumed to be finitely generated.

Proof. We give the proof only when $A$ is of equal characteristic. When $A$ is of mixed characteristic one replaces the residue fields by quasi-coefficient rings in the diagram below; these coefficient rings are formally smooth over the integers. Thus we assume that $A$ and $R$ contain the prime field of $k_{A}$. Letting $\Omega_{A}$ and $\Omega_{R}$ denote differentials over the prime field we have the exact sequence $0 \rightarrow \Gamma \rightarrow R \otimes \Omega_{A} \stackrel{h}{\rightarrow}$ $\Omega_{R} \rightarrow \Omega_{R / A} \rightarrow 0$.

(1) $\Rightarrow(2)$ : Since $k_{R}$ and $k_{A}$ are separable and hence formally smooth over the prime field [23, Th. 26.9] we have split exact sequences $0 \rightarrow \mathfrak{m}_{A} / \mathfrak{m}_{A}^{2} \rightarrow k_{A} \otimes_{A} \Omega_{A} \rightarrow$ $\Omega_{k_{A}} \rightarrow 0$ and $0 \rightarrow \mathfrak{m}_{R} / \mathfrak{m}_{R}^{2} \rightarrow k_{R} \otimes_{R} \Omega_{R} \rightarrow \Omega_{k_{R}} \rightarrow 0$ and therefore a commutative 
diagram which is exact also to the left:

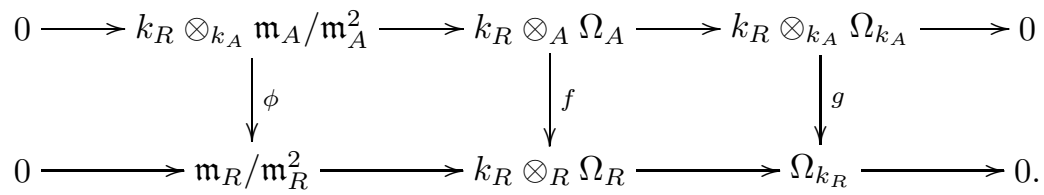

By (1) the map $f$ is surjective, hence $g$ is surjective, so $\Omega_{k_{R} / k_{A}}=0$. By Cartier's equality, using $k_{R} / k_{A}$ is finitely generated,

$$
\operatorname{rk}_{k_{R}} \Omega_{k_{R} / k_{A}}=\operatorname{tr} . \operatorname{deg}_{k_{A}} k_{R}+\operatorname{rk}_{k_{R}} \operatorname{Ker} g
$$

it follows that Ker $g=0$ and tr. $\operatorname{deg}_{k_{A}} k_{R}=0$, implying that $k_{R} / k_{A}$ is separably algebraic [loc. cit., Th. 26.6]. Therefore $g$ is an isomorphism, in particular injective; hence, as $f$ is surjective, $\phi$ is surjective by the "serpent lemma". By Nakayama's lemma it follows that $R \mathfrak{m}_{A}=\mathfrak{m}_{R}$.

$(2) \Rightarrow(1)$ : Assuming (2), the ring $R$ is a union of local $A$-algebras $F$ of finite type, $A \subset F \subset R$, such that $k_{F} / k_{A}$ is separably algebraic and $F \mathfrak{m}_{A}=\mathfrak{m}_{F}$. It is easy to see that (1) will follow if $\Omega_{F / A}=0$ for all such $F$. Replacing $R$ by $F$ in the above commutative diagram, by (2) the map $g$ is an isomorphism and $\phi$ is surjective; hence $f$ is surjective, and hence, since $\Omega_{F / A}$ is of finite type, by Nakayama's lemma, $\Omega_{F / A}=0$.

$(2) \Leftrightarrow(3)$ is evident.

If $A$ and $R$ are discrete valuation rings, then $\phi$ is an isomorphism if and only if it is injective or surjective, implying $(3) \Leftrightarrow(4)$.

The study of a ramification of a morphism $X \rightarrow Y$ can often be reduced to the case when $X$ is the spectrum of a discrete valuation ring. We now decide what a ramified morphism is in this case.

Definition 1.5.5. Let $\pi: A \rightarrow R$ be an injective homomorphism of local Nœetherian rings of the type in Proposition 1.5.1. Then $\pi$ is ramified if the two equivalent conditions (1-2) in Proposition 1.5.1 do not hold. A tamely ramified morphism $\pi$ is a ramified morphism such that the induced fraction field extension $K(R) / K(A)$ is separably algebraic, the residue field extension $k_{R} / k_{A}$ is separable, and if $x \in \mathfrak{m}_{A} \backslash \mathfrak{m}_{A}^{2}$, then $\nu(\pi(x))$ is coprime to the characteristic of $A$, where $\nu$ is the normalised valuation of $R$. A local domain $A$ of unequal characteristic with Char $k_{A}=p>0$ is (absolutely) unramified if $p \notin \mathfrak{m}_{A}^{2}$, and tamely ramified if $p \in \mathfrak{m}_{A}^{n} \backslash \mathfrak{m}_{A}^{n+1}$ for some integer $n \geq 2$ such that $p \nmid n$. A morphism of schemes $\pi: X \rightarrow Y$, where $X$ is regular in codimension $\leq 1$, is tamely ramified if for each point $x$ of height 1 in $X$, the map of local rings $\mathcal{O}_{Y, \pi(x)} \rightarrow \mathcal{O}_{X, x}$ is tamely ramified. An integral scheme $Y$ of unequal characteristic is unramified (tamely ramified) if each local ring $\mathcal{O}_{Y, y}$ is unramified (tamely ramified).

When Char $k_{A}=0$, ramified morphisms are always tamely ramified. If $R / A$ is not ramified, then we say that $R / A$ is unramified, and if a ramified morphism is not tame, then it is wild.

Remark 1.5.6. $\quad$ (i) Assuming $K(R) / K(A)$ and $k_{R} / k_{A}$ are separably finite, our definition of tameness is not the same as Abhyankar's [1, §10, p. 245] when $A$ is of mixed characteristic. 
(ii) Recall that an $A$-algebra $R$ is formally unramified if and only if $\Omega_{R / A}=0$. This is not the same as unramified in Definition 1.5.5 essentially because $\phi$ in Proposition 1.5.1 need not be injective when it is surjective. Assuming $R$ is a discrete valuation ring, by Theorem 1.5.4 and Proposition 1.5.1, it follows that if $R / A$ is unramified, then $R / A$ is formally unramified; conversely, if $R / A$ is a formally unramified extension of discrete valuation rings, and $k_{R} / k_{A}$ is finitely generated, then $R / A$ is unramified.

If $R / A$ has wild ramification we may have $T_{A / k}^{\pi} \subset \mathfrak{m}^{N} T_{A / k}$ for high $N$.

Example 1.5.7. Let $k$ be a field of positive characteristic $p$ and $\pi: A \cong R=$ $k[y] \rightarrow R=k[x]$ be a morphism over $k$. If $y=x^{n}$, and $n$ is co-prime to $p$, then $T_{A / k}^{\pi}=A y \frac{d}{d y}$. If $y=x^{p} /\left(1-x^{p-1}\right)$, then $T_{A / k}^{\pi}=A y^{2} \frac{d}{d y}$. One can iterate the latter example, letting $\pi$ be the composition $A \cong R \rightarrow R \rightarrow \cdots \rightarrow R$ ( $r$ times). This gives $T_{A / k}^{\pi}=A y^{2 r} \frac{d}{d y}$.

Lemma 1.5.8. Let $\phi_{0}: k \rightarrow k^{\prime}$ be a separably algebraic extension of fields.

(1) A homomorphism $\psi: k[[X]] \rightarrow k^{\prime}\left[\left[X^{\prime}\right]\right]$ inducing $\phi_{0}$ on residue fields is $\left(X^{\prime}\right)$-étale if and only if it is $\left(X^{\prime}\right)$-unramified if and only if $\psi(X) \in\left(X^{\prime}\right) \backslash$ $\left(X^{\prime 2}\right)$.

(2) If Char $k=p>0$, then a local homomorphism of complete p-rings $\phi$ : $(W(k), t W(k), k) \rightarrow\left(W\left(k^{\prime}\right), t^{\prime} W\left(k^{\prime}\right), k^{\prime}\right)$, extending $\phi_{0}$, is ( $\left.t^{\prime}\right)$-étale if and only it is $\left(t^{\prime}\right)$-unramified if and only if $\phi(t) \in\left(t^{\prime}\right) \backslash\left(t^{\prime 2}\right)$.

Proof. Let $R(k)$ denote $k[[t]]$ if Char $k=0$ and if Char $k=p>0$, it denotes $k[[t]]$ or the $p$-ring $W(k)$. We have a homomorphism $\phi:(R(k), t R(k), k) \rightarrow(R(k), s R(k), k)$, inducing $\phi_{0}: k \rightarrow k^{\prime}$ on residue fields. If $\phi(t) \in\left(s^{2}\right)$, the morphisms are not unramified in the $(s)$-adic topology, so it suffices to see that if $\phi(t) \in(s) \backslash\left(s^{2}\right)$, then $\phi$ is étale in the $(s)$-adic topology. Since it is clearly $(s)$-unramified it remains to see that $\phi$ is $(s)$-smooth. Since in either case $R\left(k^{\prime}\right)$ is an integral domain containing the valuation ring $R(k)$, it is flat over $R(k)$; the fibre $R\left(k^{\prime}\right) / t R\left(k^{\prime}\right)=k^{\prime}$ is separably algebraic over $k$, hence formally étale. Therefore by [13, Th. 19.7.1], $R\left(k^{\prime}\right)$ is $(s)$-étale over $R(k)$.

Remark 1.5.9. Assume that $k$ is perfect, so we have unique inclusions $W(f) \subset$ $W(g)$ over field extensions $k^{\prime} / g / f / k$, and therefore an unambiguous definition of $\lim _{k^{\prime} / f / k} W(f)$; this ring is formally étale over $W(k)$ (see argument, e.g. in [32]). However, the canonical injective homomorphism $\lim _{k^{\prime} / f / k} W(f) \rightarrow W\left(\underline{\lim }_{k^{\prime} / f / k} f\right)$ $=W\left(k^{\prime}\right)$, where the limits are over finite intermediate fields $f$, is not surjective when $k^{\prime} / k$ is not finite, and there exist no intermediate rings $\lim _{k^{\prime} / f / k} W(f) \subsetneq R \subset W\left(k^{\prime}\right)$ such that $\lim _{k^{\prime} / f / k} W(f) \rightarrow R$ is finite étale; therefore $W\left(k^{\prime}\right) / W(k)$ is not an inductive limit of finite étale morphisms. By Néron desingularisation [25] (recall also Popescu's generalisation in [26] and its new proof in [32]), formally smooth morphisms of discrete valuation rings are filtered inductive limits of finite type smooth morphisms; hence formally étale morphisms are inductive limits of (finite) étale morphisms. Therefore $W\left(k^{\prime}\right)$ is not formally étale over $W(k)$ when $k^{\prime} / k$ is not finite. Actually, letting $R(k)$ be as in the proof and the morphism $R(k) \rightarrow R\left(k^{\prime}\right)$ be $(s)$-unramified, then it is formally étale if and only if it is finite étale, and this happens if and only if $k^{\prime} / k$ is separably finite. 


\section{Liftable DERIVATIONS OF SEPARABly ALGEBRAIC MORPHiSMS}

2.1. Lifting derivations to discrete valuation rings. This section contains the main step in the description of liftable derivations. Let $\pi:\left(A, \mathfrak{m}_{A}, k_{A}\right) \rightarrow$ $\left(R, \mathfrak{m}_{R}, k_{R}\right)$ be an injective homomorphism of local Notherian algebras over a ring $k$, where $R$ is a discrete valuation ring, and assume that $A$ and $\pi$ are tamely ramified (Def. 1.5.5). In particular, derivations of $A$ are uniquely liftable to derivations of the fraction field of $R$.

Theorem 2.1.1. Let $\pi: A \rightarrow R$ be a local injective homomorphism of Notherian rings, where $R$ is a discrete valuation ring and the extension of fraction fields $K(R) / K(A)$ is separably algebraic.

(1) If $\pi$ is tamely ramified, then

$$
T_{A / k}^{\pi} \subseteq T_{A / k}\left(\log ^{\pi} K(A)\right) \cap T_{R / k}\left(\mathfrak{m}_{R}\right) \cap T_{A / k}\left(\mathfrak{m}_{A}\right) .
$$

(2) Assume that $\pi$ is residually algebraic and tamely ramified, and if $A$ is of unequal characteristic, then $A$ is tamely ramified. Then

$$
T_{A / k}^{\pi}=T_{A / k}\left(\mathfrak{m}_{A}\right)
$$

(3) If $K(A)=K(R)$, then

$$
T_{A / k}\left(\log ^{\pi} \mathfrak{m}_{A}\right) \subset T_{A / k}\left(\mathfrak{m}_{A}\right) \cap T_{R / k}\left(\mathfrak{m}_{R}\right) .
$$

(4) If $\pi$ is tamely ramified and $\mathfrak{m}_{A} \subseteq L \subseteq K(A)$, then

$$
T_{A / k}\left(\log ^{\pi} L\right)=T_{A / k}^{\pi} \cap T_{A / k}\left(\mathfrak{m}_{A}\right)=T_{A / k}^{\pi} .
$$

If moreover $\pi$ is residually algebraic and $A$ is tamely ramified (when $A$ is of unequal characteristic), then

$$
T_{A / k}^{\pi}=T_{A / k}\left(\mathfrak{m}_{A}\right)=T_{A / k}\left(\log ^{\pi} \mathfrak{m}_{A}\right) .
$$

The main part of Theorem 2.1.1 is (4) There is a certain built-in stability in this result, so that the liftable derivations do not change if $R$ is modified by a tamely ramified morphism. Let $\pi: A \stackrel{\pi_{1}}{\longrightarrow} R \stackrel{\pi_{2}}{\longrightarrow} B$, where $B$ also is a discrete valuation ring. Assuming that $\pi_{1}$ is residually algebraic and $\pi_{2}$ is tamely ramified we have

$$
T_{A / k}^{\pi}=T_{A / k}\left(\log ^{\pi_{1}} \mathfrak{m}_{A}\right) \subset T_{R / k}\left(\mathfrak{m}_{R}\right)=T_{R / k}^{\pi_{2}} \subset T_{B / k},
$$

and therefore $T_{A / k}^{\pi}=T_{A / k}^{\pi_{1}}$.

Lemma 2.1.2. Let $\left(A, \mathfrak{m}_{A}, k_{A}\right)$ be a local Notherian domain and $\partial$ a derivation of $A$. Let $\nu$ be an additive valuation on the fraction field $K(A)$ with a centre in $A$. There exists an element $c$ in the (ordered) value group of $\nu$ such that for $x \in K(A)$,

$$
\nu\left(\frac{\partial(x)}{x}\right) \geq c
$$

Proof. Select generators $\left\{x_{1}, \ldots, x_{r}\right\}$ of the maximal ideal $\mathfrak{m}_{A}$. Let $\partial$ also denote its unique extension to a derivation of $K(A)$. Let $c$ be an element in the value group satisfying $\nu\left(\partial\left(x_{i}\right) / x_{i}\right) \geq c, i=1, \ldots, r$, where we may assume $c \leq 0$. Then if $x=a b \in K(A)$, where $a, b \in K(A)$, one has $\partial(x) / x=\partial(a) / a+\partial(b) / b$; hence

$$
\nu\left(\frac{\partial(x)}{x}\right) \geq \min \left\{\nu\left(\frac{\partial(a)}{a}\right), \nu\left(\frac{\partial(b)}{b}\right)\right\} .
$$


If $a \in A$ we have $a=x_{i_{1}} x_{i_{2}} \cdots x_{i_{n}} u$ where $x_{i_{j}} \in\left\{x_{1}, x_{2}, \ldots, x_{r}\right\}$, and $u \in A \backslash \mathfrak{m}_{A}$ is a unit. Then $\nu(\partial(u) / u) \geq 0$ and by (2.1), $\nu(\partial(a) / a) \geq c$. Again by (2.1) we get for any $x=a / b \in K(A)$ that $\nu(\partial(x) / x) \geq c$.

Proof of Theorem 2.1.1. Put $p=$ Char $R$. (1): We first prove $T_{A / k}^{\pi} \subset T_{R / k}\left(\mathfrak{m}_{R}\right)$, so letting $\partial$ be a derivation of $K(A)$, hence also a derivation of $K(R)$ since $\pi$ is generically separably algebraic, such that $\partial(R) \subset R$ and $\partial(A) \subset A$, we have to see that $\partial\left(\mathfrak{m}_{R}\right) \subset \mathfrak{m}_{R}$.

Assume the contrary, that $\partial\left(\mathfrak{m}_{R}\right) \nsubseteq \mathfrak{m}_{R}$. Let $s \in \mathfrak{m}_{R} \backslash \mathfrak{m}_{R}^{2}$ be a uniformising parameter, so any element $x \in R$ is of the form $x=u s^{n}$ for a unit $u$ and positive integer $n$. From the relation

$$
\partial(x)=\left(\frac{\partial(u)}{u}+n \frac{\partial(s)}{s}\right) x
$$

and the assumption it follows that $\partial(s)$ must be a unit. Let $\nu$ be the normalised valuation of $R$, so $\nu\left(s^{n}\right)=n, \nu(\partial(s))=0$. Let $t \in \mathfrak{m}_{A} \backslash \mathfrak{m}_{A}^{2}$ have smallest possible value $n=\nu(t)$. Since by tameness $p \nmid n$, it follows that $\partial(t) \neq 0$ and $\nu(\partial(t))=n-1$; hence $\partial(t)=u$ is a unit in $A$ and $n=1$. Thus $t \in A$ is a uniformising parameter of $R$. Replacing $\partial$ by $u^{-1} \partial$ we can assume that $\partial(t)=1$. Let $\phi: R \rightarrow k_{R}$ be the projection to the residue field of $R$. We regard $k_{A}$ as a subfield of $k_{R}$, using the natural inclusion map. Let $\left\{t, x_{1}, \ldots, x_{r}\right\}$ be a subset of $\mathfrak{m}_{A}$ that induces a basis of $\mathfrak{m}_{A} / \mathfrak{m}_{A}^{2}$. Since $R / A$ is ramified we have $r \geq 1$, and writing $x_{i}=u_{i} t^{n_{i}}=u_{i} t^{\nu\left(x_{i}\right)}$, $u_{i} \in R \backslash \mathfrak{m}_{R}$, then $\phi\left(u_{i}\right) \notin k_{A}$. Consider the set

$$
S=\left\{x \in A \mid \phi\left(\frac{x}{t^{\nu(x)}}\right) \in k_{R} \backslash k_{A}\right\},
$$

which is non-empty since each $x_{i}$ belongs to $S$. If $x=u t^{n}$, for some $u \in R \backslash \mathfrak{m}_{R}$, and $p \nmid n$, by (2.2), we have $\nu(\partial(x))=n-1$ and hence $\phi\left(\partial(x) / t^{n-1}\right)=n \phi(u)$. This gives the following relation in $k_{R}$ for $x \in A$ such that $p \nmid \nu(x)$ (so in particular $\nu(x) \neq 0)$ :

$$
\phi\left(\frac{\partial(x)}{t^{\nu(\partial(x))}}\right)=\nu(x) \phi\left(\frac{x}{t^{\nu(x)}}\right) .
$$

Put $n=\nu(S)$, the minimal value of the elements in $S$. Then $n \geq 1$, for if $x \in A$ and $\nu(x)=0$, then $\phi\left(x / t^{\nu(x)}\right)=\phi(x) \in k_{A}$, so $x \notin S$. Select $x \in S$ such that $\nu(x)=n$. Then clearly $x \in \mathfrak{m}_{A} \backslash \mathfrak{m}_{A}^{2}$; hence $p \nmid \nu(x)$ since $R / A$ is tamely ramified, and hence $\nu(\partial(x))=n-1$. By (2.3) we get that $\partial(x) \in S$, contradicting the minimality of $n$. This completes the proof that $\partial\left(\mathfrak{m}_{R}\right) \subset \mathfrak{m}_{R}$.

Since $T_{A / k} \cap T_{R / k}\left(\mathfrak{m}_{R}\right)=T_{A / k}\left(\mathfrak{m}_{A}\right)$, we get $T_{A / k}^{\pi} \subset T_{A / k}\left(\mathfrak{m}_{A}\right)$. It remains to see that $T_{A / k}^{\pi} \subset T_{A / k}\left(\log ^{\pi} L\right)$ for any subset $L \subseteq K(A)$. Let $a \in L$, so $a=v t^{m}$ where $v$ is a unit in $R$, and let $\partial \in T_{A / k}^{\pi}$. We know that $\partial(t) \in R t$; hence by (2.2),

$$
\frac{\partial(a)}{a}=\frac{\partial(v)}{v}+m \frac{\partial(t)}{t} \in R .
$$

Therefore $\partial \in T_{A / k}\left(\log ^{\pi} L\right)$.

(2): Continue to regard, by generic separable algebraicity, derivations $\partial \in$ $T_{A / k}\left(\mathfrak{m}_{A}\right)$ as derivations of $K(R)$ and in particular as derivations $R \rightarrow K(R)$. We need to prove that $\partial(R) \subset R$. Let $\nu$ be the normalised valuation of $K(R)$ whose valuation ring is $R$. By Lemma 2.1.2 there exists an integer $c$ such that $\nu(\partial(r)) \geq \nu(r)-c$ for $r \in R$; hence $\partial\left(\mathfrak{m}_{R}^{n}\right) \subset \mathfrak{m}_{R}^{n-c} \subset K(R)$. Therefore, providing $K(R)$ with the linear topology where $\left\{\mathfrak{m}_{R}^{n}\right\}$ is a family of neighbourhoods of 0 , it 
follows that $\partial$ is a continuous derivation $R \rightarrow K(R)$. Hence it has a unique continuous extension to the completed rings $\partial: \bar{R} \rightarrow \lim _{n} K(R) / \mathfrak{m}_{R}^{n} \cong K(\bar{R})$; hence it has a unique continuous extension to a derivation $K(\bar{R}) \rightarrow K(\bar{R})$. By Artin-Rees' lemma, $R \subset \bar{R}$, where $\bar{R}$ also is a discrete valuation ring [6, VI, $\S 5.3$, Prop. 5]. Let $r \in R$. If $\partial(r) \notin R$, then $1 / \partial(r) \in \mathfrak{m}_{R} \subset \mathfrak{m}_{\bar{R}}$, so $\partial(r) \notin \bar{R}$. Therefore it suffices to see that $\partial(\bar{R}) \subset \bar{R}$. The map $\pi: A \rightarrow R$ induces a map $\bar{\pi}: \bar{A} \rightarrow \bar{R}$ of completed rings. Since $\bar{R}$ is integral and $\bar{A}$ is 1-dimensional (Lem. 1.4.3), Ker $\bar{\pi}$ is either $\mathfrak{m}_{\bar{A}}$ or 0 ; since $A \subset \bar{A}$ and $R \subset \bar{R}$ by Artin-Rees' lemma, it follows that $\bar{\pi}(A) \neq 0$ and therefore $\bar{\pi}$ is injective (cf. Remark 2.1.3). Since $T_{A}\left(\mathfrak{m}_{A}\right) \subset T_{\bar{A}}\left(\mathfrak{m}_{\bar{A}}\right)$ it should be clear now that one may assume that $A=\bar{A}$ and $R=\bar{R}$.

There are two cases:

(a) $A$ and $R$ are complete of unequal characteristic. Put $p=$ Char $k_{A}=$ Char $k_{R}$. The rings $A$ and $R$ contain complete $p$-rings $W\left(k_{A}\right) \subset A, W\left(k_{R}\right) \subset R$, and the field extension $k_{R} / k_{A}$ lifts to a formally unramified inclusion of $p$-rings $\left(W\left(k_{A}\right), t W\left(k_{A}\right)\right.$,

$\left.k_{A}\right) \subset\left(W^{\prime}\left(k_{R}\right), s W^{\prime}\left(k_{R}\right), k_{R}\right)$ taking $t$ to $s$ [23, Thms. 29.1-2], which is hence $\mathfrak{m}_{W^{\prime}(k)}$-étale (Lem. 1.5.8). Since all complete $p$-rings with given residue field are isomorphic [23, Cor. 29.2] we have $W^{\prime}\left(k_{R}\right) \cong W\left(k_{R}\right)$; we therefore identify $W^{\prime}\left(k_{R}\right)$ with $W\left(k_{R}\right)$, so $W\left(k_{A}\right) \rightarrow W\left(k_{R}\right) \subset R$ is $\mathfrak{m}_{W\left(k_{R}\right)}$-étale. Our derivation $\partial$ : $W\left(k_{A}\right) \rightarrow A$ induces a homomorphism of rings

$$
\phi(\partial): W\left(k_{A}\right) \rightarrow \frac{A[X]}{\left(X^{2}\right)} \subset R[X] /\left(X^{2}\right),
$$

extending the inclusion $W\left(k_{A}\right) \rightarrow R$, and by $\mathfrak{m}_{W\left(k_{R}\right)}$-étaleness it lifts uniquely to a continuous homomorphism

$$
\phi^{\prime}: W\left(k_{R}\right) \rightarrow\left(\lim _{\longleftarrow} R / \mathfrak{m}_{R}^{n}\right)[X] /\left(X^{2}\right)=\frac{R[X]}{\left(X^{2}\right)},
$$

extending the inclusion $W\left(k_{R}\right) \rightarrow R$. Such a homomorphism is of the form $\phi^{\prime}=$ $\phi\left(\partial^{\prime}\right)$ where $\partial^{\prime}$ is a derivation $W\left(k_{R}\right) \rightarrow R$. This implies

$$
\partial\left(W\left(k_{R}\right)\right)=\partial^{\prime}\left(W\left(k_{R}\right)\right) \subset R .
$$

By assumption the local ring extensions $A / W\left(k_{A}\right), R / A$ are tamely ramified, implying that $R / W\left(k_{R}\right)$ is tamely ramified. The extension $R / W\left(k_{R}\right)$ is described by the relation for a uniformising parameter $x \in \mathfrak{m}_{R} \backslash \mathfrak{m}_{R}^{2}$ : there exists an Eisenstein polynomial $f(X)=X^{n}+a_{1} X^{n-1}+\cdots+a_{n} \in W\left(k_{R}\right)[X], p \mid a_{i}, 1 \leq i \leq n, p^{2} \chi a_{n}$ (the divisibility takes place in $\left.W\left(k_{R}\right)\right)$, such that $f(x)=0$; hence $p=u x^{n}, u \in R \backslash \mathfrak{m}_{R}$. Therefore by tameness $p \nmid n$. Applying $\partial$ gives $f^{\prime}(x) \partial(x)+f^{\partial}(x)=0$, where $f^{\partial}(x)=$ $x^{n-1} \partial\left(a_{1}\right)+\cdots+\partial\left(a_{n}\right)$. Since $\partial\left(a_{i}\right) \in R$ and $\partial(p)=0$ we have $\partial\left(a_{i}\right)=r_{i} p=r_{i}^{\prime} x^{n}$, for some $r_{i}, r_{i}^{\prime} \in R$, and it follows that one can write $f^{\partial}(x)=r x^{n}$, for some $r \in R$. Since $p \nmid n$ we have $f^{\prime}(x)=n x^{n-1}+(n-1) a_{1} x^{n-2}+\cdots+a_{n-1}=u x^{n-1}$, where $u \in R \backslash \mathfrak{m}_{R}$. It follows that $\partial(x)=-f^{\partial}(x) / f^{\prime}(x)=-r x / u \in \mathfrak{m}_{R} \subset R$.

(b) $A$ and $R$ are complete of equal characteristic. Then $A$ has a coefficient field $k_{A} \subset A$ which injects into $R$ and this image is part of a coefficient field $k_{R}$ of $R$, so $k_{A} \subset k_{R} \subset R=k_{R}[[t]]$, where $t \in \mathfrak{m}_{R} \backslash \mathfrak{m}_{R}^{2}$. Since $k_{R} / k_{A}$ is separably algebraic there exists to each $\alpha \in k_{R}$ a polynomial $f \in k_{A}[X]$ such that $f(\alpha)=0, f^{\prime}(\alpha) \neq 0$. Applying $\partial$ gives $f^{\prime}(\alpha) \partial(\alpha)+f^{\partial}(\alpha)=0$; hence $\partial(\alpha) \in k_{R} A \subset R$. This implies

$$
\partial\left(k_{R}\right) \subset R .
$$


It remains to see that $\partial(t) \in R t$. Assume the contrary, $\partial(t) \notin(t)$. Choose $x \in A$ such that $R \mathfrak{m}_{A}=R x$, and write $x=u t^{n}$, where $u$ is a unit in $R$. Since $\partial\left(k_{R}\right) \subset R$ we have $\nu(\partial(u)) \geq \nu(\partial(t))$. Therefore, since $n$ and $p$ are coprime, $\nu(\partial(x))<\nu(x)$. This contradicts the assumptions $\partial(x) \in \mathfrak{m}_{A}$ and $\nu\left(\mathfrak{m}_{A}\right)=\nu(x)$.

(3): Let $\partial \in T_{A / k}\left(\log ^{\pi} \mathfrak{m}_{A}\right)$. Then for $a \in A$ we have $\partial(a)=r a \in A$ for some $r \in R$. Any element $z \in K(A)$ can be written $a / b$ with $a, b \in \mathfrak{m}_{A}$, implying

$$
\frac{\partial(z)}{z}=\frac{\partial(a)}{a}-\frac{\partial(b)}{b} \in R .
$$

Since $K(R)=K(A)$ it follows that $\partial(R) \in R$ and $\partial\left(\mathfrak{m}_{R}\right) \subset \mathfrak{m}_{R}$; moreover $T_{A / k}^{\pi} \cap$ $T_{R / k}\left(\mathfrak{m}_{R}\right) \subset T_{A / k}\left(\mathfrak{m}_{A}\right)$. (When $R / A$ is tamely ramified the inclusion is an equality by (1).)

(4): By (1), $T_{A / k}^{\pi} \subset T_{R / k}\left(\mathfrak{m}_{R}\right) \cap T_{A / k}\left(\log ^{\pi} \mathfrak{m}_{A}\right) \subset T_{A / k}\left(\mathfrak{m}_{A}\right)$, so it remains to see that $T_{A / k}\left(\log ^{\pi} \mathfrak{m}_{A}\right) \subset T_{A / k}^{\pi}$. Put $R_{1}=R \cap K(A)$, which again is a discrete valuation ring and $k_{R} / k_{R_{1}}$ is algebraic (Lem. 1.4.3). We have inclusions of local rings

$$
A \stackrel{\pi_{1}}{\hookrightarrow} R_{1} \stackrel{i}{\hookrightarrow} R,
$$

so $\pi=i \circ \pi_{1}$. That $\pi$ is tamely ramified implies that $\pi_{1}$ and $i$ are tamely ramified. Let $\partial \in T_{A / k}\left(\log ^{\pi} \mathfrak{m}_{A}\right)$. Then for $a \in A$ we have $\partial(a)=r a \in A$ for some $r \in R$; hence $r \in R \cap K(A)=R_{1}$, and hence $\partial(a) \in R_{1} a$. Therefore

$$
T_{A / k}\left(\log ^{\pi} \mathfrak{m}_{A}\right) \subset T_{A / k}\left(\log ^{\pi_{1}} \mathfrak{m}_{A}\right) \subset T_{A / k}\left(\mathfrak{m}_{A}\right) \cap T_{R_{1} / k}\left(\mathfrak{m}_{R_{1}}\right),
$$

where the last inclusion follows from (3). In particular, $T_{A / k}\left(\log ^{\pi} \mathfrak{m}_{A}\right) \subset$ $T_{R_{1} / k}\left(\mathfrak{m}_{R_{1}}\right)$. Since $i$ is tamely ramified and $k_{R} / k_{R_{1}}$ is algebraic, by (2) we have $T_{R_{1} / k}\left(\mathfrak{m}_{R_{1}}\right) \subset T_{R_{1} / k}^{i} ;$ therefore $T_{A / k}\left(\log ^{\pi} \mathfrak{m}_{A}\right) \subset T_{A / k}^{\pi}$.

Remark 2.1.3. If $A \rightarrow R$ is a local injective map of Notherian rings, it does not follow that the induced map of completed rings $\bar{\pi}: \bar{A} \rightarrow \bar{R}$ is injective (see [10,17]) but derivations of $A$ extend to derivations of $\bar{A}$ that preserve the kernel of $\bar{\pi}$, since the identity $\pi \circ \partial-\partial \circ \pi=0$ extends, by continuity, to the identity $\bar{\pi} \circ \partial=\partial \circ \bar{\pi}$.

\subsection{Proof of the lifting theorem.}

Theorem 2.2.1. Let $\pi: X / S \rightarrow Y / S$ be a morphism of schemes over a scheme $S$. Assume that $Y$ and $S$ are Notherian and $X$ is a Krull scheme. Assume that $\pi$ is dominant, generically algebraic and tamely ramified (Def. 1.5.5). If $Y$ is of unequal characteristic, assume that $Y$ is absolutely tamely ramified. Let $D_{\pi}$ be the discriminant set of $\pi$. Then:

$\left(L_{1}\right)$ Let $J_{D_{\pi}}$ be any defining ideal of the closure of $D_{\pi}$. Then

$$
T_{Y / S}^{\pi}=T_{Y / S}\left(\log ^{\pi} J_{D_{\pi}}\right) .
$$

$\left(L_{2}\right)$ If the residue field extension $k_{c} / k_{\pi(c)}$ is algebraic for each point $c \in C_{\pi}$ of height 1 , then

$$
T_{Y / S}^{\pi}=T_{Y / S}\left(I_{D_{\pi}}\right)
$$

where $I_{D_{\pi}}$ is the (reduced) ideal of the closure of the discriminant set $D_{\pi}$.

$\left(L_{3}\right)$ Let $I_{C_{\pi}}$ be the ideal of the critical locus of $\pi$. Then $\pi^{-1}\left(T_{Y / S}^{\pi}\right) \subset T_{X / S}\left(I_{C_{\pi}}\right)$; i.e., the liftable vector fields are tangent to the critical locus. 
Proof. Since $X / Y$ is generically separably algebraic any derivation $\partial$ of $\mathcal{O}_{Y}(U)$, where $U$ is open in $Y$, has a unique lift $\bar{\partial}$ to a derivation of the field $\mathcal{O}_{X, \xi}$ over the generic point $\xi$ of $X$. Since $X$ is Krull it suffices to see that $\bar{\partial}\left(\mathcal{O}_{X, c}\right) \subset \mathcal{O}_{X, c}$ when $c \in X$ is a point of height 1 that maps to $U$, for then $\bar{\partial}\left(\mathcal{O}_{\pi^{-1}(U)}\right) \subset \mathcal{O}_{\pi^{-1}(U)}$. If $c \notin C_{\pi}$, i.e. $d \pi_{c}$ is surjective, this is evidently the case, so assume $c \in C_{\pi}$ is a point of height 1 in $X$. Let $c$ be a point of $C_{\pi}$ of height 1 . Put $R=\mathcal{O}_{X, c}, A=\mathcal{O}_{Y, \pi(c)}$, so we may regard $A$ as a subring of the discrete valuation ring $R$, with inclusion morphism $\pi: A \rightarrow R$. Put also $k=\mathcal{O}_{S, s}$. Let $\mathfrak{m}_{A}$ and $\mathfrak{m}_{R}$ be the maximal ideals of $A$ and $R$, so $\mathfrak{m}_{A} \subset \mathfrak{m}_{R}$. We need to prove that $T_{A / k}^{\pi}=T_{A / k}\left(\log ^{\pi} \mathfrak{m}_{A}\right)$, and if $k_{R} / k_{A}$ is algebraic, $T_{A / k}^{\pi}=T_{A / k}\left(\mathfrak{m}_{A}\right)$. By Proposition 1.5.1, (1) $\Rightarrow(3)$, and the morphism $A \rightarrow R$ is ramified (hence tamely ramified). Therefore $\left(L_{1}\right)$ follows from Theorem 2.1.1, (3), and $\left(L_{2}\right)$ follows from Theorem 2.1.1, (2). The assertion in $\left(L_{3}\right)$ follows from Theorem 2.1.1 (1).

2.3. The normalisation morphism. Let $\pi: \bar{X} / S \rightarrow X / S$ be the normalisation of an integral Nœetherian scheme.

Theorem 2.3.1. (1) $T_{X / S}\left(\log ^{\pi} I_{D}\right) \subset T_{X / S}^{\pi}$.

(2) If the normalisation morphism $\pi$ is tamely ramified, then $T_{X / S}^{\pi}=T_{X / S} \subset$ $T_{\bar{X} / S}\left(I_{C}\right)$ (in particular, $\pi$ is weakly submersive).

Proof. To alleviate the notation slightly we consider only the case $S=\operatorname{Spec} \mathbf{Z}$; the general relative case is no different. Let $x \in \bar{X}, y=\pi(x)$ and put $A=\mathcal{O}_{x}, B=\mathcal{O}_{y}$, where $y$ lies over a prime number $s$. We have an inclusion of integral domains $A \subset B$ with coinciding fraction fields, $K=K(A)=K(B)$, and $B$ is integrally closed in $K$. We need to prove that a derivation $\partial: A \rightarrow A$ can be lifted to a derivation $\tilde{\partial}: B \rightarrow B$. First, $\partial$ can be extended uniquely to a derivation $\tilde{\partial}: K \rightarrow K$. By the Mori-Nagata theorem [24, Th. 33.10] the normalisation of a Nœtherian integral domain is a Krull ring; hence $B$ is the intersection of the discrete valuation rings $R$ in $K$ containing $B$. Hence we may regard $A$ as a subring of a discrete valuation ring $R$ and it suffices to see that $\tilde{\partial}(R) \subset R$. Localise $A$ at the prime $p \in \operatorname{Spec} B$ such that $R=B_{p}$. Then $A_{p} \subset R$ and $\partial\left(A_{p}\right) \subset A_{p}$, so one may assume $A=A_{p}$. We have then an algebraic extension $\left(A, \mathfrak{m}_{A}, k_{A}\right) \rightarrow\left(R, \mathfrak{m}_{R}, k_{R}\right)$ of one-dimensional local rings where $R$ is regular. Let $\nu$ be the normalised discrete valuation of $R$. If $A=R$ there is nothing to prove; hence one may assume that $A$ is not regular (i.e. not normal).

(1): Let $\partial \in T_{A}\left(\log ^{\pi} \mathfrak{m}_{A}\right)$. The proof that $\partial(R) \subset R$ is similar to the proof of (3) in Theorem 2.1.1. Any element in $K(A)=K(R)$ can be written $z=a / b$ with $a, b \in \mathfrak{m}_{A}$. Since $\partial(a) \in R a$ for each $a \in \mathfrak{m}_{A}$, we get

$$
\frac{\partial(z)}{z}=\frac{\partial(a)}{a}-\frac{\partial(b)}{b} \in R
$$

In particular, $\partial(R) \subset R$. This proves $T_{A}\left(\log ^{\pi} \mathfrak{m}_{A}\right) \subset T_{A}^{\pi}$.

(2): Let $\partial$ be a derivation of $A$, inducing a derivation of $K(A)=K(R)$, and reasoning as in the proof of Theorem 2.1.1. (2), it induces a derivation $\bar{R} \rightarrow K(\bar{R})$; we have $A \subset R \subset \bar{R}$. Since $\bar{R} \cap K(R)=R$ it suffices to see that $\partial(\bar{R}) \subset \bar{R}$. Let $t \in \mathfrak{m}_{\bar{R}} \backslash \mathfrak{m}_{\bar{R}}^{2}$ be a uniformising parameter. Let $W\left(k_{R}\right)$ either be a complete $p$-ring such that $\bar{R}$ is an Eisenstein extension of $W\left(k_{R}\right)$, or a coefficient field in $\bar{R}$, and let $W\left(k_{A}\right)$ either be a complete $p$-ring or a coefficient field in $A$ such that $W\left(k_{A}\right) \subset W\left(k_{R}\right)$. Since $B / A$ is integral, so the localisation $A_{q} \subset B_{p}=R$ is integral, 
where $p$ is a prime ideal in $B$ and $q=A \cap p$. By assumption the field extension $k_{R} / k_{A_{q}}$ is separably algebraic. We can assume that $A=A_{q}$. Now follow the proof of (2) in Theorem 2.1.1. First we get $\partial\left(W\left(k_{R}\right)\right) \subset R$, which is a consequence of the fact that $W\left(k_{R}\right) / W\left(k_{A}\right)$ is $\mathfrak{m}_{W\left(k_{R}\right)}$-étale. Note that this follows without a priori assuming $\partial\left(\mathfrak{m}_{A}\right) \subset \mathfrak{m}_{A}$. Secondly, we get $\partial(\bar{R}) \subset \bar{R}$ if $\partial(t) \in \bar{R}$. Finally, by Theorem 2.1.1. (1), if $T_{A}=T_{A}^{\pi}$, it follows that $T_{A}=T_{A}\left(\mathfrak{m}_{A}\right) \subset T_{R}\left(\mathfrak{m}_{R}\right)$.

Assume the contrary, that $\nu(\partial(t))<0$, where $\nu$ is the normalised valuation of $\bar{R}$. Let $x_{1}, \ldots, x_{n}$ be a system of parameters of $\mathfrak{m}_{A}$ and put $m_{i}=\nu\left(x_{i}\right)$, where we may assume $m_{1} \leq m_{2} \leq \cdots \leq m_{n}$. Since $p \nmid m_{i}$, we get

$$
\nu\left(\partial\left(x_{i}\right)\right)=\nu\left(x_{i}\right)+\nu\left(\frac{\partial\left(u_{i}\right)}{u_{i}}+m_{i} \frac{\partial(t)}{t}\right)=m_{i}+\nu(\partial(t))-1,
$$

where $x_{i}=u_{i} t^{m_{i}}$ for some unit $u_{i} \in R$. The last equality follows since $\partial\left(W\left(k_{R}\right)\right) \subset$ $R$, implying $\nu(\partial(u)) \geq \nu(\partial(t))$ (since $\nu(\partial(t))<0$ ); this holds both in equal and mixed characteristic. Since $\partial\left(x_{1}\right) \in A$ and $\nu(\partial(t))<0$, so $0 \leq m_{1}+\nu(\partial(t))-1<$ $m_{1}$, we get $\nu(\partial(t))=1-m_{1}$ and $m_{1} \geq 2$. To get a contradiction, it suffices to see that $m_{1} \mid m_{i}, i=1,2, \ldots, n$, since then $\mathbf{Z} m_{1}+\mathbf{Z} m_{2}+\cdots+\mathbf{Z} m_{n}$ is a proper ideal of $\mathbf{Z}$ which would be in opposition to $K(A)=K(R)$. Since $m_{1} \mid m_{1}$, assume $m_{1} \mid m_{i}$ when $i=1,2, \ldots, r-1$ for some integer $r \geq 2$. We get from (2.4),

$$
\nu\left(\partial\left(x_{r}\right)\right)=m_{r}-m_{1}=\sum_{i=1}^{r-1} a_{i} m_{i},
$$

where $a_{i} \in \mathbf{N}$; the last equality follows since $\nu\left(\partial\left(x_{r}\right)\right)<m_{r}$. Hence $m_{1} \mid m_{r}$.

Corollary 2.3.2. Let $\left(A, \mathfrak{m}_{A}\right)$ be a local 1-dimensional integral Noetherian ring with a tamely ramified normalisation. If there exists a derivation $\partial$ of $A$ and an element $x \in \mathfrak{m}_{A} \backslash \mathfrak{m}_{A}^{2}$ such that $\partial(x) \notin \mathfrak{m}_{A}$, then $A$ is regular.

Proof. From the above proof we get $T_{A}=T_{A}\left(\mathfrak{m}_{A}\right)$ if $A$ is 1-dimensional and not normal, implying the assertion.

Remark 2.3.3. Corollary 2.3.2 follows from Zariski's lemma when $A$ contains the rational numbers and its completion is reduced (see [23, Corollary to Th. 30.1]). A more instructive argument that $T_{A / k}=T_{A / k}\left(\mathfrak{m}_{A}\right)$ for singular one-dimensional $k$-algebras $A$ can be achieved in a special case. Assume that $A$ is a local ring such that the Jacobian criterion of regularity holds. Then the Jacobian ideal $J$ is preserved by $T_{A / k}$, i.e. $T_{A / k}=T_{A / k}(J)$. If $A$ is of characteristic 0 , we have $T_{A / k}=T_{A / k}(\sqrt{J})$ (see e.g. [19]); hence if $A$ is singular, we have a proper $T_{A / k^{-}}$ invariant radical ideal $\sqrt{J} \subset \mathfrak{m}_{A}$. Therefore, if $A$ is a singular one-dimensional ring it follows that $T_{A / k}=T_{A / k}\left(\mathfrak{m}_{A}\right)$. In Corollary 2.3.2 no assumption is made on the characteristic or the validity of the Jacobian criterion. However, for wildly ramified normalisations the existence of $\partial \in T_{A / k}$ and $t \in \mathfrak{m}_{A}$ such that $\partial(t)=1$ does not imply that $A$ is regular (Ex. 2.3.5).

Remark 2.3.4. What Seidenberg actually proved is this. Let $A$ be an integral domain, containing the rational numbers $\mathbf{Q}, \bar{A}$ its integral closure and $A^{\prime}$ the ring of quasi-integral elements (so $\bar{A}=A^{\prime}$ when $A$ is Notherian). Then $T_{A / \mathbf{Q}} \subset T_{A^{\prime} / \mathbf{Q}}$, and if the ring of formal power series $\bar{A}[[t]]$ is integrally closed, then $T_{A / \mathbf{Q}} \subset T_{\bar{A} / \mathbf{Q}}$. We say that a derivation $\partial$ of $A$ is integrable if there exists a Hasse-Schmidt derivation of the form $\left(1, \partial, D_{2}, \ldots\right)$. Note that the higher derivations $D_{i}$ are not uniquely 
determined by $\partial$; see 22 . The idea in Seidenberg's proof is to consider only the integrable derivations, giving rise to automorphisms of $A[[t]]$, and the point is that derivations are always integrable to higher derivations when $\mathbf{Q} \subset A$. On the other hand, if $\mathbf{Q} \nsubseteq A A$ the question of integrability is a delicate matter. The only general condition I am aware of that all derivations of a $k$-algebra $A$ be integrable is that $A / k$ be smooth 22 , but this is of no interest for the lifting problem, at least when $k$ is a perfect field, since then $A$ is normal. Letting $T_{X / S}^{i n t}$ be the sheaf of integrable derivations, Seidenberg's theorem states that $T_{X / S}^{i n t} \subset T_{X / S}^{\pi}$ when $\pi$ is the normalisation morphism of a Nœtherian scheme [30] (also when $\pi$ is wildly ramified). Assume that $X / k$ is a variety. Matsumura [22 proves that if $k$ is a perfect field, then $T_{X / k}^{i n t}$ is of the same rank as $T_{X / k}$, and gives an example that over an imperfect field $k$ one may have $T_{X / k}^{i n t}=0$ while $T_{X / k} \neq 0$. On the other hand, $T_{X / S}\left(\log ^{\pi} \mathfrak{m}_{A}\right) \subset T_{X / k}^{\pi}$ (Th. 2.3.1), and this module is always non-zero when $T_{X / k} \neq 0$.

We extend an example by Seidenberg [30] giving many examples of rings with wild normalisations and where there exist derivations that do not lift.

Example 2.3.5. Let $R$ be a discrete valuation ring of characteristic $p>0, K$ its fraction field and $\partial$ a derivation of $R$ such that $\partial\left(\mathfrak{m}_{R}\right) \not \subset \mathfrak{m}_{R}$; we also regard $\partial$ as a derivation of $K$ and let $K^{\partial} \subset K$ be the subfield of elements $r$ such that $\partial(r)=0$ (so $R^{p} \subset K^{p} \subset K^{\partial}$ ). Suppose $S$ is a subset of $R$ such that $\partial(S) \subset S$ and $\partial^{n}(S)=0$ for some positive integer $n$. The set $\mathfrak{m}_{R}^{\partial}=K^{\partial} \cap \mathfrak{m}_{R}$ is non-trivial if and only if Char $R=p>0$, and then $\mathfrak{m}_{R}^{\partial} \subset \mathfrak{m}_{R}^{p}$. Select an element $c \in \mathfrak{m}_{R}^{\partial}$ and an increasing sequence of subsets $B_{0} \subset B_{1} \subset \cdots \subset B_{n} \subset \mathfrak{m}_{R}^{\partial}$ such that $B_{i} \subset c B_{i+1}$ and let $A$ be the smallest local subring of $R$ that contains the set

$$
S_{B}=S B_{0}+\partial(S) B_{1}+\cdots+\partial^{n-1}(S) B_{n} .
$$

Since clearly $\frac{1}{c} \partial\left(S_{B}\right) \subset S_{B}$ we get $\frac{1}{c} \partial(A) \subset A$, but $\frac{1}{c} \partial(R) \not \subset R$. If $S$ generates $K$ and $S \cap B_{0} \neq \emptyset$, then $K(A)=K$ and the inclusion $\pi: A \rightarrow R$ is the normalisation of $A$. The derivation $\delta=\frac{1}{c} \partial$ of $A$ does not belong to $T_{A}\left(\log ^{\pi} \mathfrak{m}_{A}\right)$. Let $k$ be a field of characteristic $p, R=k[t]_{0}, S=\left\{t^{p}, t^{p+1}\right\} \subset k[t]_{0}$ and $B_{i}=t^{(i+1) p}$. Then $A=k\left[t^{p}, t^{p+1}\right]_{0} \subset R=k[t]_{0} \subset k(t)$, which is an extension with ramification index $p$. This is close to Seidenberg's example. Then $\delta=t^{-p} \partial_{t}$ is a derivation of $A$ such that $\delta(R) \not \subset R$. Clearly, $\delta\left(t^{p+1}\right) \notin R t^{p+1}$, so $\delta \notin T_{A}\left(\log ^{\pi} \mathfrak{m}_{A}\right)$. Note also that $\partial_{t} \in T_{A}^{\pi}$, but $\partial_{t}\left(\mathfrak{m}_{R}\right) \not \subset \mathfrak{m}_{R}$, showing that (1) in Theorem 2.1.1 need not hold when $\pi$ is wildly ramified.

All derivations may lift even if the normalisation is wildly ramified.

Example 2.3.6. Put $A=k\left[t^{3}, t^{7}, t^{8}\right]_{0} \subset R=k[t]_{0}$ and assume Char $k=3$. Here $T_{A / k}=A \partial_{t} \subset T_{R / k}$.

\section{Birational MORPhisms}

3.1. Differentially ramified morphisms. Assuming the morphism $\pi$ is of the type in Theorem 2.2.1 we have $T_{X / Y}=0$. Therefore, if $J$ is an ideal of $\mathcal{O}_{X}$ it makes sense to define the sub-sheaf of $T_{X / S}$ of liftable derivations that preserve $J$ by

$$
T_{Y / S}^{\pi}(J)=T_{Y / S}^{\pi} \cap T_{X / S}(J) .
$$


For an ideal $I$ of $\mathcal{O}_{Y}$, its inverse image ideal is $\tilde{I}=\mathcal{O}_{X} I$ (with obvious interpretation).

Definition 3.1.1. A dominant morphism of schemes $\pi: X \rightarrow Y$, where $X$ is Krull and $Y$ is locally Notherian, with discriminant locus $D$, is uniformly ramified at a generic point $\xi$ of the critical locus $C$ if the stalk $I_{D, \pi(\xi)}$ of the ideal of $D$ at $\pi(\xi)$ has a basis $I_{D, \pi(\xi)}=\left(x_{1}, \ldots, x_{l}\right)$ satisfying $\nu_{\xi}\left(x_{i}\right)=\nu_{\xi}\left(x_{j}\right)$, for each $i, j$. The morphism $\pi$ is uniformly ramified if it is uniformly ramified at each generic point of the critical locus $C$.

Thus the condition is on the base $Y$ but local at each generic point of $C$. Do not confuse this with another notion of uniform ramification in [11.

Definition 3.1.2. A generically finite morphism $\pi$ is differentially ramified if

$$
T_{Y / S}^{\pi}=T_{Y / S}\left(I_{D}\right),
$$

where $I_{D}$ is the (reduced) ideal of of the discriminant $D$.

Thus for differentially ramified morphisms the liftable derivations can be characterised in terms of the discriminant $D$ only, and do not depend on the particular differentially ramified morphism with such a discriminant. For example, residually algebraic morphisms as in Theorem 2.2.1 are differentially ramified.

Theorem 3.1.3. Let $\pi: X \rightarrow Y$ be a morphism as in Theorem 2.2.1,

(1) If $\pi$ is uniformly ramified, then it is differentially ramified.

(2) Assume that for each generic point $\xi$ of $C$, there exists a basis $I_{D, \pi(\xi)}=$ $\left(x_{1}, \ldots, x_{r}\right)$ and derivations $\partial_{1}, \ldots, \partial_{r} \in T_{Y / S, \pi(\xi)}$ such that $\operatorname{det} \partial_{j}\left(x_{i}\right)$ is invertible. Then $\pi$ is uniformly ramified if and only if it is differentially ramified.

For example, by (1), if $D_{\pi}$ is a hypersurface, i.e. $I_{D_{\pi}}$ is locally principal, then morphisms of the type considered are differentially ramified.

Proof. (1): If $\pi$ is uniformly ramified a derivation $\partial \in T_{Y / S}\left(I_{D}\right)$ satisfies, for each generic point $\xi$ of $C, \nu_{\xi}\left(\partial\left(x_{i}\right)\right) \geq \nu_{\xi}\left(x_{i}\right)$ for some basis $\left(x_{1}, \ldots, x_{l}\right)$ of $I_{D, \pi(\xi)}$. The assertion therefore follows from Theorem 2.2.1.

(2): By (1) we have only to prove that $\pi$ is uniformly ramified when $T_{Y / S}^{\pi}=$ $T_{Y / S}\left(I_{D}\right)$. Let $c_{i j}$ be the inverse matrix of $\left(\partial_{i}\left(x_{j}\right)\right)$. Setting $\partial_{i}^{\prime}=\sum c_{i j} \partial_{j}$ we have $\partial_{i}^{\prime}\left(x_{j}\right)=\delta_{i j}$, so that we can assume $\partial_{i}\left(x_{j}\right)=\delta_{i j}$. By assumption $T_{Y / S}\left(I_{D}\right) \subset$ $T_{Y / S}\left(\log ^{\pi} I_{D}\right)$ (Th. 2.2.1); hence $\nu_{\xi}\left(x_{i}\right)=\nu_{\xi}\left(x_{i} \partial_{j}\left(x_{j}\right)\right) \geq \nu_{\xi}\left(x_{j}\right)$. By symmetry the opposite inequality also holds.

3.2. Blow-ups. A projective birational morphism of integral Nœtherian schemes $\pi: \tilde{X} \rightarrow X$ can be constructed as a blow-up of a coherent fractional ideal on $X$, and if $X$ and $Y$ are projective over a field, one may even construct $\pi$ as a blowup of an ideal [15, Prop. 2.3.5, Cor. 2.3.7]. Let $X$ be an integral scheme with sheaf of rational functions $R_{X}$, let $I$ be a coherent $\mathcal{O}_{X}$-submodule of $R_{X}$, and let $\pi: B l_{I}(X) \rightarrow X$ be the blow-up of $X$ along $I$. We will in this section try to describe the liftable derivations $T_{X / S}^{\pi}$, without using valuations along the critical locus of $\pi$; more precisely, we look for a (fractional) ideal $J$ such that $T_{X / S}^{\pi}=T_{X / S}(J)$. Clearly, if such an ideal exists, then $D_{\pi}=\left\{x \in X \mid J_{x} \neq \mathcal{O}_{X, x}\right\}$, the discriminant locus of $\pi$, so $J$ and $I$ would have the same radical. 
Let $p: \overline{B l_{I}(X)} \rightarrow B l_{I}(X)$ be the normalisation morphism of the blow-up of $I$. The integral closure of $I$ is the sheaf $\bar{I}$ whose section over an open set $U$ is

$$
\bar{I}(U)=\bigcap_{x \in U, \operatorname{ht}(x) \leq 1} \mathcal{O}_{\overline{B l_{I}(X)}, x} I_{x} .
$$

Lemma 3.2.1 (see [33]).

$$
\begin{aligned}
\bar{I}(U)= & \left\{f \in R_{X}(U) \mid \text { there exists an equation } f^{n}+b_{1} f^{n-1}+\cdots+b_{n}=0,\right. \\
& \left.n>0, b_{i} \in I^{i}(U)\right\} \\
= & (\pi \circ p)_{*}\left(I \mathcal{O}_{\overline{B l_{I}(X)}}\right) .
\end{aligned}
$$

Lemma 3.2.1 implies that $\bar{I}$ is coherent since $\pi \circ p$ is proper. Clearly, $I \subset \bar{I}$, and if $X$ is normal and $I$ is an ideal of $\mathcal{O}_{X}$, then $\bar{I} \subset \mathcal{O}_{X}$. See [16] for relations between $\overline{B l_{I}(X)}$ and $B l_{I^{n}}(X)$ for high $n$.

Define $\left[I^{n+1}: I^{n}\right]=\left\{a \in R_{X} \mid a I^{n} \subset I^{n+1}\right\}$; this is a coherent fractional ideal. Then for sufficiently high $n$ we have $\left[I^{n+2}: I^{n+1}\right]=\left[I^{n+1}: I^{n}\right]$. Assuming that $I$ is coherent, the Ratliff-Rush fractional ideal $\hat{I}$ associated to $I$ is the common fractional ideal $\left[I^{n+1}: I^{n}\right]$ for high $n$, and we have $\hat{I} \subset \bar{I}$. One has $I^{n}=(\hat{I})^{n}$ for high $n$ [loc. cit.]. These assertions are well known when $I$ is a coherent ideal 27, and they immediately generalise to fractional ideals since a coherent fractional ideal is locally contained in an ideal of the form $J / f$, where $J$ is a coherent ideal of $\mathcal{O}_{X}$, and we have $\left[I^{n+1}: I^{n}\right]=\frac{1}{f}\left[(f I)^{n+1}:(f I)^{n}\right]$.

Theorem 3.2.2. Let $X / S$ be an integral Notherian scheme with sheaf of rational functions $R_{X}$. Let $I$ be a coherent $\mathcal{O}_{X}$-submodule of $R_{X}$ and $\pi: B l_{I}(X) \rightarrow X$ be the blow-up of $X$ over $I$.

(1) $T_{X / S}(\hat{I}) \subset T_{X / S}^{\pi} \subset T_{X / S}(\bar{I})$ (the right inclusion holds when the normalisation morphism $p$ is tamely ramified).

(2) $T_{X / S}(I) \subset T_{X / S}^{\pi} \cap T_{X / S}(\bar{I}) \cap T_{X / S}(\hat{I})$.

Assume that $p$ is tamely ramified. If $\hat{I}=\bar{I}$ (e.g. $I=\bar{I}$ ), then $T_{X / S}^{\pi}=T_{X / S}(\bar{I})$. If $I$ is radical, then $\pi$ is differentially ramified.

Proof. We first prove $T_{X / S}(I) \subset T_{X / S}^{\pi}$. A derivation that preserves $I$ extends to a derivation of the Rees algebra $\bigoplus_{n \geq 0} I^{n}$, and therefore induces a derivation of each affine chart $B_{(f)}=\bigcup_{j \geq 0} I^{j} / f^{j}, f \in I$, of $B l_{I}(X)=\operatorname{Proj} \bigoplus_{n \geq 0} I^{n}$. Since in such a chart $\tilde{I}=B I(=f B)$, clearly $\partial(\tilde{I}) \subset \tilde{I}$.

$T_{X / S}^{\pi} \subset T_{X / S}(\bar{I})$ : We have $T_{X / S}^{\pi} \subset T_{X / S}^{\pi \circ p}$ since $p$ is tamely ramified (Th. 2.3.1). As $\overline{B l_{I}(X)}$ is Krull, by Theorem 2.2.1, $\left(L_{3}\right)$, a liftable derivation $\partial$ lifts to a derivation $\tilde{\partial} T_{\overline{B l_{I}(X)} / S}$ that preserves the ideal of the critical divisor. Since $\tilde{I}=I \mathcal{O}_{\overline{B l_{I}(X)}}$ is a locally principal ideal defining the critical locus of $\pi \circ p$, it follows that $\tilde{\partial}(\tilde{I}) \subset \tilde{I}$. Therefore $\partial$ preserves $(\pi \circ p)_{*}(\tilde{I})=\bar{I}$.

$T_{X / S}(\hat{I}) \subset T_{X / S}^{\pi}:$ Let $\hat{\pi}: B l_{\hat{I}}(X) \rightarrow X$ be the blow-up of $\hat{I}$. We then have

$$
T_{X / S}(\hat{I}) \subset T_{X / S}^{\hat{\pi}}=T_{X / S}^{\pi},
$$

where the latter equality follows since $\hat{I}^{n}=I^{n}$ when $n \gg 1$, so $B l_{\hat{I}}(X)=B l_{I}(X)$. This completes the proof of the assertions (1) and (2) 
Assume $\hat{I}=\bar{I}$, which holds in particular when $I$ is radical. Then $(1)$ implies $T_{X / S}^{\pi}=T_{X / S}(\bar{I})$, which in particular implies that $\pi$ is differentially ramified when $I$ is radical.

Corollary 3.2.3. Let $\pi: \tilde{X} / S \rightarrow X / S$ be a tamely ramified blow-up of a reduced subscheme $V / S$ of an integral Noetherian scheme $X / S$, such that $\tilde{X}$ is Krull. If $X / S$ is smooth at generic points of $V$, then $\pi$ is uniformly ramified.

Suppose $s$ is a point in $S$ with perfect residue field and that the fibre $V_{s}$ is a smooth subscheme of $X_{s}$. Then $\tilde{X}_{s}$ is regular and hence Krull (normal).

Proof. For each generic point of the critical locus its image $x$ is a generic point of $V \subset X$. Let $k$ be the residue field of the image $s$ of $x$ in $S$. The canonical exact sequence $0 \rightarrow \mathfrak{m}_{X, x} / \mathfrak{m}_{X, x}^{2} \rightarrow k_{X, x} \otimes \Omega_{X / S, x} \rightarrow \Omega_{k_{X, x} / k} \rightarrow 0$ is (split) exact since $k_{X, x} / k$ is formally smooth. Assume that $\left\{x_{1}, \ldots, x_{r}\right\} \subset \mathfrak{m}_{X, x}$ induces a basis $\left\{\bar{x}_{1}, \ldots, \bar{x}_{r}\right\}$ of $\mathfrak{m}_{X, x} / \mathfrak{m}_{X, x}^{2}$, so $\mathfrak{m}_{X, x}=\left(x_{1}, \ldots, x_{r}\right)$, and select elements $\omega_{i} \in \Omega_{X / S, x}$ such that $\left\{x_{1}, \ldots, x_{r}, \omega_{1}, \ldots, \omega_{s}\right\}$ induces a basis $\left\{1 \otimes d_{X / S}\left(x_{1}\right), \ldots, 1 \otimes d_{X / S}\left(x_{r}\right), 1 \otimes\right.$ $\left.\omega_{1}, \ldots, 1 \otimes \omega_{s}\right\}$ of $k_{X, x} \otimes \Omega_{X / S, x}$. Since $\Omega_{X / S, x}$ is a free $\mathcal{O}_{X, x}$-module of finite type it follows that $\left\{d_{X / S}\left(x_{1}\right), \ldots, d_{X / S}\left(x_{r}\right), \omega_{1}, \ldots, \omega_{s}\right\}$ are free generators of $\Omega_{X / S, x}$; hence there exist derivations $\partial_{i} \in T_{X / S, x}$ satisfying $\partial_{i}\left(x_{j}\right)=\delta_{i j}$. Since $V$ is reduced and $\pi$ is tamely ramified it follows that $\pi$ is differentially ramified (Th. 3.2.2), so the assertion can be concluded from Theorem 3.1.3, (2).

For an ideal $I$ of a polynomial ring $R$ over a field it is in general a laborious task to compute its integral closure $\bar{I}[33$, although for monomial ideals it is simply the monomial ideal defined by the convex hull of the exponent vectors of $I$. The RatliffRush ideal associated with $I$ seems even more difficult to compute (see [7,28]); for instance, I am unaware of any simple description of $\hat{I}$ for monomial $I$. Notice that to compute the liftable derivations $T_{X / S}^{\pi}$ for a blow-up using Theorem 2.2.1 we need to know that $B l_{I}(X)$ is normal. This however is difficult to read off from $I$ (a sufficient condition is that $I^{n}$ be integrally closed for high $n$ ).

Example 3.2.4. Let $R=\mathbf{Q}\left[\left[t^{4}, t^{5}, t^{6}, t^{7}\right]\right]$ (singular ring) and $I=\left(t^{4}, t^{5}\right)$. Then $I^{2}=\left(t^{8}, t^{9}, t^{10}\right),\left[I^{2}: I\right]=I,\left[I^{3}: I^{2}\right]=\left(t^{4}, t^{5}, t^{6}, t^{7}\right)=\mathfrak{m}=\hat{I}$. We have $T_{R / \mathbf{Q}}(I)=R t \partial_{t}$ and $T_{R / \mathbf{Q}}(\hat{I})=R t \partial t+R t^{2} \partial_{t}$. Hence $T_{R / \mathbf{Q}}(\hat{I}) \neq T_{R / \mathbf{Q}}(I)$.

Example 3.2.5. Let $R=\mathbf{Q}[x, y]_{(x, y)}$ (regular ring), $I=\left(x^{10}, x^{8} y, x y^{4}, y^{5}\right)$. Then $\hat{I}=\left(x^{10}, y^{5}, x y^{4}, x^{7} y^{2}, x^{6} y^{3}, x^{8} y\right)([7,28])$ and $\bar{I}=\left(x^{10}, x^{8} y, x^{6} y^{2}, x^{4} y^{3}, x y^{4}, y^{5}\right)$. Generators of the module of derivations of a monomial $\mathfrak{m}$-primary ideal $J=$ $\left(x^{a_{i}} y^{b_{i}}\right)_{i=0}^{r}, a_{i}>a_{i+1}$ are easily computed. Let $h=\max \left(a_{i}-a_{i+1}\right)$ and $w=$ $\max \left(b_{i+1}-b_{i}\right)$. Then $T_{R}(J)=R x \partial_{x}+R y \partial_{y}+R y^{h} \partial_{x}+R x^{w} \partial_{y}$. In particular, $T_{R / \mathbf{Q}}(I)=\left(x, y^{3}\right) \partial_{x}+\left(x^{7}, y\right) \partial_{y}, T_{R / \mathbf{Q}}(\hat{I})=(x, y) \partial_{x}+\left(x^{5}, y\right) \partial_{y}$, and $T_{R / \mathbf{Q}}(\bar{I})=$ $(x, y) \partial_{x}+\left(x^{3}, y\right) \partial_{y}$, so $T_{R / \mathbf{Q}}(I) \neq T_{R / \mathbf{Q}}(\hat{I}) \neq T_{R / \mathbf{Q}}(\bar{I})$. Consider the morphism

$$
\pi: B l_{I}(\operatorname{Spec} R) \rightarrow \operatorname{Spec} R .
$$

Since $\hat{I} \neq \bar{I}$ it follows that $B l_{I}(\operatorname{Spec} R)$ cannot be normal, so we cannot use Theorem 2.2 .1 to compute $T_{R / \mathbf{Q}}^{\pi}$; we know only that $(x, y) \partial_{x}+\left(x^{5}, y\right) \partial_{y} \subset T_{R / \mathbf{Q}}^{\pi} \subset$ $(x, y) \partial_{x}+\left(x^{3}, y\right) \partial_{y}$. It would require some effort to determine whether the derivations $x^{3} \partial_{y}, x^{4} \partial_{y}$ belong to $T_{R / \mathbf{Q}}^{\pi}$. 
Remark 3.2.6. We have $T_{X / S}(I) \subset T_{X / S}\left(I^{n}\right) \subset T_{X / S}\left(\left[I^{n}: I^{n-1}\right]\right)$, but in general $T_{X / S}\left(I^{n}\right) \not \subset T_{X / S}(I)$, when $I \neq \hat{I}$, as seen from Example 3.2.4 for $n=3$.

We have $T_{X / S}(I) \subset T_{X / S}(\hat{I}) \subset T_{X / S}(\bar{I})$ where the first inclusion follows from Remark 3.2.6. while the latter, valid when $p$ is tamely ramified, follows from Theorem 3.2.2, that these inclusions in general are strict is shown in Example 3.2.5, One can refine these inclusions. In [31, Shah defines for $\mathfrak{m}$-primary ideals in a local ring the notion of coefficient ideals, using coefficients of the Hilbert polynomial of $I$; this notion was extended in [16. Def. 3.21] to general ideals $I$ in a Nœetherian domain, defining coefficient ideals as contractions of pull-backs of $I$ by certain birational morphisms. Let $I$ be a coherent ideal of $\mathcal{O}_{X}$, where $X$ is an integral and Nœetherian scheme of dimension $d$, and let $I_{\{k\}}, k=0, \ldots, d$ be the coefficient ideals of $I$ as defined in [loc. cit.]; put also $I_{\{d+1\}}=I$. One has $I \subset I_{\{d\}}=\hat{I} \subset I_{\{d-1\}} \subset \cdots \subset I_{\{1\}} \subset I_{\{0\}}=\bar{I}$ [loc. cit] and one can prove, as in the beginning of the proof of Theorem 3.2.2 noting that the lifted derivations evidently preserve the intersections of local rings described in [loc. cit, 3.14], that $T_{X / S}(I) \subset T_{X / S}\left(I_{\{d\}}\right)=T_{X / S}(\hat{I}) \subset T_{X / S}\left(I_{\{d-1\}}\right) \subset \cdots \subset T_{X / S}\left(I_{\{k\}}\right) \subset \cdots \subset$ $T_{X / S}\left(I_{\{1\}}\right) \subset T_{X / S}(\bar{I})$, at least if the birational map that is used to define $I_{\{k\}}$ is tamely ramified. Let $J_{\{k\}}$ be the smallest coherent ideal that contains $I_{\{k\}}$ such that $T_{X / S}\left(I_{\{k-1\}}\right) \subset T_{X / S}\left(J_{\{k\}}\right)$. We then have

$$
I_{\{k\}} \subset J_{\{k\}} \subset I_{\{k-1\}},
$$

and one may wonder when these inclusions are strict? In Examples 3.2 .6 and 3.2 .5 we have $J_{\{d+1\}}=I_{\{d\}}(=\hat{I})$. If one knows a priori that $J_{\{k\}}=I_{\{k-1\}}$, one can compute $I_{\{k-1\}}$ from a knowledge of $I_{\{k\}}$ and $T_{X / S}\left(I_{\{k-1\}}\right)$, since $J_{\{k\}}=$ $\mathcal{D}_{X / S}\left(T_{X / S}\left(I_{\{k-1\}}\right)\right) I_{\{k\}}$ where the ring $\mathcal{D}_{X / S}\left(T_{X / S}\left(I_{\{k-1\}}\right)\right)$ of differential operators is generated by $T_{X / S}\left(I_{\{k-1\}}\right)$. However, one cannot use Theorem 2.2.1 to describe $T_{X / S}\left(I_{\{k-1\}}\right)$, since the source of the birational morphisms used to define $I_{\{k-1\}}$ is not Krull.

We end this paper with two concrete illustrations of the use of Theorems 2.1.1 and 3.2 .2 .

Example 3.2.7. Let $A$ be the polynomial ring $k\left[y_{1}, \ldots, y_{r}\right]$ localised at some prime ideal, and assume that $A \subset R$ is tamely ramified and $K(R) / K(A)$ is finite, where $R$ is a discrete valuation ring with valuation $\nu$. Theorem 2.1.1 implies $\partial=a_{1} \partial_{y_{1}}+$ $\cdots+a_{r} \partial_{y_{r}} \in T_{A / k}^{\pi}, a_{i} \in A$, if and only if $\nu\left(a_{i}\right) \geq \nu\left(y_{i}\right), i=1, \ldots, r$. To be very explicit, let $A=k\left[y_{1}, y_{2}\right]_{\left(y_{1}, y_{2}\right)}, R=k\left[x_{1}, x_{2}\right]_{\left(x_{1}\right)}$, so $\mathfrak{m}_{A}=\left(y_{1}, y_{2}\right)$ and $\mathfrak{m}_{R}=\left(x_{1}\right)$, and define a birational map $\pi: A \rightarrow R$ by $y_{1}=x_{1}, y_{2}=x_{1}^{n} x_{2}$, where $n$ is an integer $\geq 1$. Then $\partial_{y_{1}}=\partial_{x_{1}}-\left(n x_{2} / x_{1}\right) \partial_{x_{2}}$ and $\partial_{y_{2}}=x_{1}^{-n} \partial_{x_{2}}$, acting on $K(R)=k\left(x_{1}, x_{2}\right)$. The condition $\partial \in T_{A / k}\left(\log ^{\pi} \mathfrak{m}_{A}\right)$ is that $\partial \in T_{A / k}$ and $\nu\left(\partial\left(y_{1}\right)\right) \geq \nu\left(y_{1}\right)=1$, $\nu\left(\partial\left(y_{2}\right)\right) \geq \nu\left(y_{2}\right)$, i.e. $a_{1} \in\left(y_{1}, y_{2}\right)$ and $a_{2} \in\left(y_{1}^{n}, y_{2}\right)$; therefore $T_{A / k}\left(\log ^{\pi} \mathfrak{m}_{A}\right)=$ $\mathfrak{m}_{A} \partial_{y_{1}}+\left(y_{1}^{n}, y_{2}\right) \partial_{y_{2}}$. If Char $k=0$ or $n$ is coprime to Char $k$, by Theorem 2.1.1]

$$
T_{A / k}^{\pi}=T_{A / k}\left(\log ^{\pi} \mathfrak{m}_{A}\right)=\mathfrak{m}_{A} \partial_{y_{1}}+\left(y_{1}^{n}, y_{2}\right) \partial_{y_{2}} .
$$

We have then

$$
R T_{A / k}^{\pi}=R \nabla_{1}+R \partial_{x_{2}}=T_{R / k}\left(I_{C_{\pi}}\right),
$$

where the critical ideal $I_{C_{\pi}}$ is $\left(x_{1}\right)$. If $n=1$, so $\pi$ comes by a chart of the blowup of $\mathfrak{m}_{A}$, then $T_{A / k}^{\pi}=T_{A / k}\left(\log ^{\pi} \mathfrak{m}_{A}\right)=\mathfrak{m}_{A} \partial_{y_{1}}+\mathfrak{m}_{A} \partial_{y_{2}}=T_{A / k}\left(\mathfrak{m}_{A}\right)$, so $\pi$ is differentially ramified, in agreement with Theorem 3.2.2, noting that $\mathfrak{m}_{A}$ is radical. 
If $n \geq 2$, corresponding to a successive blowing up of points, then the derivations $y_{1}^{k} \partial_{y_{2}} \in T_{A / k}\left(\mathfrak{m}_{A}\right), k=1, \ldots, n-1$ are not liftable. In this case $A \rightarrow R$ comes by a chart of the blow-up of the ideal $I=\left(y_{1}^{n}, y_{2}\right)$; this ideal is Ratliff-Rush and integrally closed: $I=\hat{I}=\bar{I}$. It is clear that $T_{A / k}^{\pi}=\mathfrak{m}_{A} \partial_{y_{1}}+\left(y_{1}^{n}, y_{2}\right) \partial_{y_{2}}=T_{A / k}(I)$, as predicted by Theorem 3.2.2. If Char $k=p>0$ and $p$ divides $n$, so $\pi$ has wild ramification, then $T_{A / k}^{\pi}=A \partial_{y_{1}}+\left(y_{1}^{n}, y_{2}\right) \partial_{y_{2}}$.

Example 3.2.8. Put $B=\mathbf{Q}[x, s, t] /\left(s^{4}-x t\right)$ and $A=\mathbf{Q}[x, y]$ and define the homomorphism $\pi: A \rightarrow B, \pi(x)=x, \pi(y)=x^{2} s$. The critical divisor is given by the principal ideal $(s) \subset B$ and $x=s^{4} / t$ in $B_{(s)}$, so the corresponding valuation $\nu: A \rightarrow \mathbf{Z}$ is determined by $\nu(x)=4, \nu(y)=9$. A derivation $a \partial_{x}+b \partial_{y} \in T_{A / \mathbf{Q}}$ lifts to a derivation of $B$ if $\nu(a) \geq \nu(x)=4$ and $\nu(b) \geq \nu(y)=9$ (Th.2.1.1), noting that $B$ is a normal ring, implying $T_{A / \mathbf{Q}}^{\pi}=(x, y) \partial_{x}+\left(x^{2}, y\right) \partial_{y}$.

\section{REFERENCES}

[1] S. S. Abhyankar, Resolution of singularities of embedded algebraic surfaces, 2nd ed., Springer Monographs in Mathematics, Springer-Verlag, Berlin, 1998. MR1617523 (99c:14021)

[2] V. I. Arnol'd, Wave front evolution and equivariant Morse lemma, Comm. Pure Appl. Math. 29 (1976), no. 6, 557-582. MR55:9148

[3] M. Auslander and D. A. Buchsbaum, On ramification theory in noetherian rings, Amer. J. Math. 81 (1959), 749-765. MR0106929 (21:5659)

[4] Edward Bierstone and Pierre D. Milman, Canonical desingularization in characteristic zero by blowing up the maximum strata of a local invariant, Invent. Math. 128 (1997), no. 2, 207-302. MR1440306 (98e:14010)

[5] A. Borel, P.-P. Grivel, B. Kaup, A. Haefliger, B. Malgrange, and F. Ehlers, Algebraic Dmodules, Perspectives in Mathematics, vol. 2, Academic Press, Inc., Boston, MA, 1987. MR882000 (89g:32014)

[6] Nicolas Bourbaki, Elements of Mathematics. Commutative algebra, Hermann, Paris, 1972. MR0360549 (50:12997)

[7] Juan Elias, On the computation of the Ratliff-Rush closure, J. Symbolic Comput. 37 (2004), no. 6, 717-725. MR2095368 (2005j:13022)

[8] S. Encinas and O. Villamayor, Good points and constructive resolution of singularities, Acta Math. 181 (1998), no. 1, 109-158. MR1654779(99i:14020)

[9] Robert M. Fossum, The divisor class group of a Krull domain, Springer-Verlag, New York, 1973. Ergebnisse der Mathematik und ihrer Grenzgebiete, Band 74. MR 0382254 (52:3139)

[10] A. M. Gabrièlov, Formal relations among analytic functions, Izv. Akad. Nauk SSSR Ser. Mat. 37 (1973), 1056-1090 (Russian). MR0346184 (49:10910)

[11] Phillip Griffith, Some results in local rings on ramification in low codimension, J. Algebra 137 (1991), no. 2, 473-490. MR1094253 (92c:13017)

[12] A. Grothendieck, Éléments de géométrie algébrique. I. Le langage des schémas, Inst. Hautes Études Sci. Publ. Math. 4 (1960), 228. MR0217083(36:177a)

[13] _ Éléments de géométrie algébrique. IV. Étude locale des schémas et des morphismes de schémas. I, Inst. Hautes Études Sci. Publ. Math. 20 (1964), 259 (French). MR0173675 $(30: 3885)$

[14] A Grothendieck, Éléments de géométrie algébrique. IV. Étude locale des schémas et des morphismes de schémas IV, Inst. Hautes Études Sci. Publ. Math. 32 (1967), 361 (French). MR0238860 (39:220)

[15] Alexander Grothendieck and Jean Dieudonné, Étude cohomologique des faisceaux cohérents, Publ. IHES 4 (1961).

[16] William Heinzer, Bernard Johnston, David Lantz, and Kishor Shah, Coefficient ideals in and blowups of a commutative Noetherian domain, J. Algebra 162 (1993), no. 2, 355-391. MR:1254782 (95b:13020)

[17] Reinhold Hübl, Completions of local morphisms and valuations, Math. Z. 236 (2001), no. 1, 201-214. MR1812456(2002d:13003) 
[18] Rolf Källström, Purity of branch, critical, and discriminant locus, 2006. Submitted, available at arXiv:math/0609300.

[19] _ Preservation of defect sub-schemes by the action of the tangent sheaf, J. Pure and Applied Algebra 203 (2005), no. 1-3, 166-188. MR2176658 (2006g:14002)

[20] Robert Lazarsfeld, Positivity in algebraic geometry. II, Ergebnisse der Mathematik und ihrer Grenzgebiete. 3. Folge. A Series of Modern Surveys in Mathematics [Results in Mathematics and Related Areas. 3rd Series. A Series of Modern Surveys in Mathematics], vol. 49, Springer-Verlag, Berlin, 2004. Positivity for vector bundles, and multiplier ideals. MR 2095472 (2005k:14001b)

[21] Joseph Lipman, Equisingularity and simultaneous resolution of singularities, Resolution of singularities (Obergurgl, 1997), Progr. Math., vol. 181, Birkhäuser, Basel, 2000, pp. 485-505. MR:1748631 (2001e:32044)

[22] Hideyuki Matsumura, Integrable derivations, Nagoya Math. J. 87 (1982), 227-245. MR0676593 (84e:13007)

[23] Commutative ring theory, Cambridge University Press, 1986. MR879273 (88h:13001)

[24] Masayoshi Nagata, Local rings, Robert E. Krieger Publishing Co., Huntington, N.Y., 1975. Corrected reprint. MR0460307 (57:301)

[25] André Néron, Modèles minimaux des variétés abéliennes sur les corps locaux et globaux, Inst. Hautes Études Sci. Publ. Math. No. 21 (1964), 128 (French). MR0179172 (31 \#3423)

[26] Dorin Popescu, General Néron desingularization and approximation, Nagoya Math. J. 104 (1986), 85-115. MR868439 (88a:14007)

[27] L. J. Ratliff Jr. and David E. Rush, Two notes on reductions of ideals, Indiana Univ. Math. J. 27 (1978), no. 6, 929-934. MR0506202 (58:22034)

[28] Maria Evelina Rossi and Irena Swanson, Notes on the behavior of the Ratliff-Rush filtration, Commutative algebra (Grenoble/Lyon, 2001), 2003, pp. 313-328. MR2013172 (2005b:13006)

[29] Günther Scheja and Uwe Storch, Fortsetzung von Derivationen, J. Algebra 54 (1978), no. 2, 353-365 (German). MR514074 (80a:13004)

[30] A Seidenberg, Derivations and integral closure, Pacific J. Math. 16 (1966), 167-173. MR0188247 (32:5686)

[31] Kishor Shah, Coefficient ideals, Trans. Amer. Math. Soc. 327 (1991), no. 1, 373-384. MR1013338 (91m:13008)

[32] Mark Spivakovsky, A new proof of D. Popescu's theorem on smoothing of ring homomorphisms, J. Amer. Math. Soc. 12 (1999), no. 2, 381-444. MR.1647069 (99j:13008)

[33] Wolmer Vasconcelos, Integral closure, Rees algebras, multiplicities, algorithms, Springer Monographs in Mathematics, Springer-Verlag, Berlin, 2005. MR2153889 (2006m:13007)

[34] Orlando Villamayor U., On equiresolution and a question of Zariski, Acta Math. 185 (2000), no. 1, 123-159. MR.1794188 (2002a:14003)

[35] Orlando Villamayor, Constructiveness of Hironaka's resolution, Ann. Sci. École Norm. Sup. (4) 22 (1989), no. 1, 1-32. MR985852 (90b:14014)

[36] Oscar Zariski, Studies in equisingularity. I. Equivalent singularities of plane algebroid curves, Amer. J. Math. 87 (1965), 507-536.MR 31 \#2243, MR0177985 (31:2243)

Department of Mathematics, University of Gävle, 80176 Gävle, Sweden

E-mail address: rkm@ig.se 